\title{
Sharp Convergence Rates for Darcy's Law
}

\author{
Zhongwei Shen*
}

\begin{abstract}
This paper is concerned with Darcy's law for an incompressible viscous fluid flowing in a porous medium. We establish the sharp $O(\sqrt{\varepsilon})$ convergence rate in a periodically perforated and bounded domain in $\mathbb{R}^{d}$ for $d \geq 2$, where $\varepsilon$ represents the size of solid obstacles. This is achieved by constructing two boundary layer correctors to control the boundary layers created by the incompressibility condition and the discrepancy of boundary values between the solution and the leading term in its asymptotic expansion. One of the correctors deals with the tangential boundary data, while the other handles the normal boundary data.
\end{abstract}

Keywords: Darcy's Law; Convergence Rate; Stokes Equations.

MR (2020) Subject Classification: 35Q35; 35B27; 76D07.

\section{Introduction}

This paper is concerned with Darcy's law for an incompressible viscous fluid in a porous medium. More precisely, we consider the Dirichlet problem for the steady Stokes equations,

$$
\left\{\begin{aligned}
-\varepsilon^{2} \mu \Delta u_{\varepsilon}+\nabla p_{\varepsilon}=f & \text { in } \Omega_{\varepsilon}, \\
\operatorname{div}\left(u_{\varepsilon}\right)=0 & \text { in } \Omega_{\varepsilon}, \\
u_{\varepsilon}=0 & \text { on } \partial \Omega_{\varepsilon},
\end{aligned}\right.
$$

where $\mu>0$ is the viscosity constant, $0<\varepsilon<1$, and $\Omega_{\varepsilon}$ is a periodically perforated and bounded domain in $\mathbb{R}^{d}, d \geq 2$. In (1.1) we have normalized the velocity vector by a factor $\varepsilon^{2}$, where $\varepsilon$ is the period. To describe the porous domain $\Omega_{\varepsilon}$, we let $Y=[0,1]^{d}$ be a closed unit cube and $Y_{s}$ (solid part) an open subset of $Y$ with Lipschitz boundary. Throughout the paper we shall assume that $\operatorname{dist}\left(\partial Y, \partial Y_{s}\right)>0$ and that $Y_{f}=Y \backslash \overline{Y_{s}}$ (the fluid part) is connected. Let $\Omega$ be a bounded domain in $\mathbb{R}^{d}$ with Lipschitz boundary. For $0<\varepsilon<1$, define

$$
\Omega_{\varepsilon}=\Omega \backslash \bigcup_{k} \varepsilon\left(\overline{Y_{s}}+z_{k}\right),
$$

where $z_{k} \in \mathbb{Z}^{d}$ and the union is taken over those $k$ 's for which $\varepsilon\left(Y+z_{k}\right) \subset \Omega$.

For $f \in L^{2}\left(\Omega ; \mathbb{R}^{d}\right)$, let $\left(u_{\varepsilon}, p_{\varepsilon}\right) \in H_{0}^{1}\left(\Omega_{\varepsilon} ; \mathbb{R}^{d}\right) \times L^{2}\left(\Omega_{\varepsilon}\right)$ be the weak solution of (1.1) with $\int_{\Omega_{\varepsilon}} p_{\varepsilon} d x=0$. We extend $u_{\varepsilon}$ to the whole domain $\Omega$ by zero and still denote the extension by $u_{\varepsilon}$. Let $P_{\varepsilon}$ be the extension of $p_{\varepsilon}$ to $\Omega$, defined by (2.6). It has been known since late 1970's that as $\varepsilon \rightarrow 0, u_{\varepsilon} \rightarrow u_{0}$ weakly in $L^{2}\left(\Omega ; \mathbb{R}^{d}\right)$ and $P_{\varepsilon} \rightarrow p_{0}$ strongly in $L^{2}(\Omega)$, where $\left(u_{0}, p_{0}\right)$ is given by a Darcy law,

$$
\begin{cases}u_{0}=\mu^{-1} K\left(f-\nabla p_{0}\right) & \text { in } \Omega, \\ \operatorname{div}\left(u_{0}\right)=0 & \text { in } \Omega \\ u_{0} \cdot n=0 & \text { on } \partial \Omega\end{cases}
$$

${ }^{*}$ Supported in part by NSF grant DMS-1856235 and by Simons Fellowship. 
with $\int_{\Omega} p_{0} d x=0$. In (1.3) the permeability matrix $K=\left(K_{j}^{i}\right)$ is a $d \times d$ positive definite and symmetric matrix defined by (2.3), and $n$ denotes the outward unit normal to $\partial \Omega$. Furthermore, it was observed in [3] by G. Allaire that as $\varepsilon \rightarrow 0$,

$$
u_{\varepsilon}-\mu^{-1} W(x / \varepsilon)\left(f-\nabla p_{0}\right) \rightarrow 0 \quad \text { strongly in } L^{2}\left(\Omega ; \mathbb{R}^{d}\right)
$$

where $W(y)=\left(W_{j}^{i}(y)\right)$ is an 1-periodic $d \times d$ matrix defined by the cell problem (2.2) and $f_{Y} W(y) d y=K$. For an excellent exposition on Darcy's law and closely related topics, we refer the reader to [4] by G. Allaire and A. Mikelić.

The purpose of this paper is to study the convergence rates for $u_{\varepsilon}-\mu^{-1} W(x / \varepsilon)\left(f-\nabla p_{0}\right)$ and $P_{\varepsilon}-p_{0}$ in $L^{2}(\Omega)$. The following is the main result of the paper. The $O(\sqrt{\varepsilon})$ rate in (1.5) is sharp.

Theorem 1.1. Let $\Omega$ be a bounded $C^{2, \alpha}$ domain in $\mathbb{R}^{d}, d \geq 2$ for some $\alpha>0$. Also assume that $Y_{s}$ is an open subset of $Y=[0,1]^{d}$ with $C^{1, \alpha}$ boundary. Let $\left(u_{\varepsilon}, p_{\varepsilon}\right) \in H_{0}^{1}\left(\Omega_{\varepsilon} ; \mathbb{R}^{d}\right) \times L^{2}\left(\Omega_{\varepsilon}\right)$ be a weak solution of (1.1), where $f \in C^{1,1 / 2}\left(\bar{\Omega} ; \mathbb{R}^{d}\right)$ and $\int_{\Omega_{\varepsilon}} p_{\varepsilon} d x=0$. Then

$$
\begin{aligned}
\left\|u_{\varepsilon}-\mu^{-1} W(x / \varepsilon)\left(f-\nabla p_{0}\right)\right\|_{L^{2}(\Omega)}+\left\|P_{\varepsilon}-p_{0}\right\|_{L^{2}(\Omega)} \\
+\left\|\varepsilon \nabla u_{\varepsilon}-\mu^{-1} \nabla W(x / \varepsilon)\left(f-\nabla p_{0}\right)\right\|_{L^{2}(\Omega)} \leq C \sqrt{\varepsilon}\|f\|_{C^{1,1 / 2}(\Omega)},
\end{aligned}
$$

where $C$ depends only on $d, \mu, \Omega$, and $Y_{s}$.

The first rigorous proof of Darcy's law by homogenization was given by L. Tartar in an appendix of [23], using an energy method. We refer the reader to [4] for references on earlier work on the formal derivation of Darcy's law, using two-scale asymptotic expansions. In [2, 3], the strong convergence of $\left(u_{\varepsilon}, P_{\varepsilon}\right)$ in $L^{2}(\Omega)$ was established by the method of two-scale convergence. Also see related work in [15, 20, 8, 17, 18, 19, 16].

Regarding the rate of convergence for $\left(u_{\varepsilon}, P_{\varepsilon}\right)$ in $L^{2}(\Omega)$, to the best of the author's knowledge, the only previous result for a bounded domain with the Dirichlet condition was obtained by E. Marušić-Paloka and A. Mikelić in [17], where a rate $O\left(\varepsilon^{1 / 6}\right)$ was established for the case $d=2$. See [8] for an earlier result for a unbounded domain $\Omega=(0, L) \times \mathbb{R}_{+}$. We remark that for Laplace's equation and systems of linear elasticity, quantitative error estimates have been established in [14, 11, 22, 10, 9]. As pointed out in [17], the simple cut-off argument, which seems to work well for standard elliptic equations and systems, does not yield any convergence rate for the Stokes equations because of the incompressibility condition. In [17], using a stream function from [24], a boundary layer corrector was constructed in the case $d=2$ to control the boundary layer near $\partial \Omega$ created by the incompressibility condition. We mention that [17] also treated the case of nonlinear stationary Navier-Stokes equations.

We now describe our approach to the problem of convergence rates and error estimates, which is based on energy estimates. Let

$$
u(x, x / \varepsilon)=\mu^{-1} W(x / \varepsilon)\left(f(x)-\nabla p_{0}(x)\right) .
$$

To address the discrepancy of boundary values between $u_{\varepsilon}$ and $u(x, x / \varepsilon)$ as well as the incompressibility condition, we introduce two boundary layer correctors $\left(\Psi_{t}, q_{t}\right)$ and $\left(\Psi_{n}, q_{n}\right)$. Let $\partial \Omega_{\varepsilon}=\partial \Omega \cup \Gamma_{\varepsilon}$. The tangential boundary layer corrector $\left(\Psi_{t}, q_{t}\right)$ is a weak solution of

$$
\left\{\begin{aligned}
-\varepsilon^{2} \mu \Delta \Psi_{t}+\nabla q_{t}=0 & \text { in } \Omega_{\varepsilon}, \\
\operatorname{div}\left(\Psi_{t}\right)=0 & \text { in } \Omega_{\varepsilon},
\end{aligned}\right.
$$

with boundary data $\Psi_{t}=0$ on $\Gamma_{\varepsilon}$, and

$$
\Psi_{t}=-u(x, x / \varepsilon)+[u(x, x / \varepsilon) \cdot n] n \quad \text { on } \partial \Omega .
$$


Note that $\Psi_{t} \cdot n=0$ on $\partial \Omega$. By the divergence theorem and the Cauchy inequality, this gives,

$$
\left\|\nabla \Psi_{t}\right\|_{L^{2}\left(\Omega_{\varepsilon}\right)}^{2} \leq\left\|\nabla \Psi_{t}\right\|_{L^{2}(\partial \Omega)}\left\|\Psi_{t}\right\|_{L^{2}(\partial \Omega)} .
$$

We use a localized Rellich estimate in a Lipschitz domain to show that

$$
\left\|\nabla \Psi_{t}\right\|_{L^{2}(\partial \Omega)} \leq C\left\{\left\|\nabla_{\tan } \Psi_{t}\right\|_{L^{2}(\partial \Omega)}+\varepsilon^{-1 / 2}\left\|\nabla \Psi_{t}\right\|_{L^{2}\left(\Omega_{\varepsilon}\right)}\right\},
$$

where $\nabla_{\tan } \Psi_{t}$ denotes the tangential gradient of $\Psi_{t}$ on the boundary $\partial \Omega$. The desired $O(\sqrt{\varepsilon})$ bound for $\varepsilon\left\|\nabla \Psi_{t}\right\|_{L^{2}\left(\Omega_{\varepsilon}\right)}$ follows from (1.9) and (1.10). See Section 4 for details.

The normal boundary layer corrector $\left(\Psi_{n}, q_{n}\right)$ is defined as the solution of the Stokes equations (1.7) in $\Omega_{\varepsilon}$, with the boundary conditions $\Psi_{n}=0$ on $\Gamma_{\varepsilon}$, and

$$
\Psi_{n}=-[u(x, x / \varepsilon) \cdot n-\gamma] n \quad \text { on } \partial \Omega,
$$

where

$$
\gamma=f_{\partial \Omega} u(x, x / \varepsilon) \cdot n d \sigma
$$

Thanks to (1.3), we may write

$$
u(x, x / \varepsilon) \cdot n=\mu^{-1} n_{i}\left[W_{j}^{i}(x / \varepsilon)-K_{j}^{i}\right]\left(f_{j}-\frac{\partial p_{0}}{\partial x_{j}}\right) \quad \text { on } \partial \Omega
$$

(the repeated indices are summed from 1 to $d$ ). Furthermore, there exists a 1-periodic tensor $\left(\phi_{\ell j}^{i}\right)$ such that

$$
\phi_{\ell j}^{i}=-\phi_{i j}^{\ell} \quad \text { and } \quad W_{j}^{i}(y)-K_{j}^{i}=\frac{\partial}{\partial y_{\ell}} \phi_{\ell j}^{i}(y) .
$$

It follows from (1.12) and (1.13) that

$$
u(x, x / \varepsilon) \cdot n=\varepsilon(2 \mu)^{-1}\left(n_{i} \frac{\partial}{\partial x_{\ell}}-n_{\ell} \frac{\partial}{\partial x_{i}}\right)\left(\phi_{\ell j}^{i}(x / \varepsilon)\right) \cdot\left(f_{j}-\frac{\partial p_{0}}{\partial x_{j}}\right) \quad \text { on } \partial \Omega .
$$

Since $n_{i} \frac{\partial}{\partial x_{\ell}}-n_{\ell} \frac{\partial}{\partial x_{i}}$ is a tangential derivative, the formula (1.14) allows us to use an integration by parts on $\partial \Omega$ (see (5.21)), which generates the needed decay factor $\varepsilon$. In order to carry out this argument, we use an energy estimate to reduce the problem to the $L^{2}$ estimate for the Stokes equations in $\Omega$, whose solutions are then represented by integrals on $\partial \Omega$, using the Poisson kernels. See Section 5 for details.

We point out that the $C^{1, \alpha}$ condition on $Y_{s}$ in Theorem 1.1 is used to ensure the boundedness of $\nabla W$, while the $C^{2, \alpha}$ condition on $\Omega$ is used for the $C^{2}$ estimates for the Stokes equations in $\Omega$. The $C^{1,1 / 2}$ condition on $f$ seems to be more or less optimal for the methods used. An $O(\sqrt{\varepsilon})$ estimate with less regularity on $f$ would be an interesting and challenging problem.

The paper is organized as follows. In Section 2 we introduce some notations and collect several known results that will be used in later sections. In Section 3 we establish an energy estimate for the Stokes equations in $\Omega_{\varepsilon}$. The tangential boundary layer corrector $\left(\Psi_{t}, q_{t}\right)$ is constructed in Section 4, while the normal boundary layer corrector $\left(\Psi_{n}, q_{n}\right)$ and its estimates are given in Section 5. The proof of Theorem 1.1 is contained in Section [6, where an interior corrector is constructed. In fact, a more general case is treated in Section 6, where we assume $u_{\varepsilon}=b \in H^{1}\left(\partial \Omega ; \mathbb{R}^{d}\right)$ on $\partial \Omega$. See Theorem 6.1. Due to the discrepancy of $u_{\varepsilon}$ and $u(x, x / \varepsilon)$ on $\partial \Omega$, the $O(\sqrt{\varepsilon})$ rate in Theorem 1.1 is sharp. See Remark 6.6

Throughout the paper, the repeated indices are summed from 1 to $d$. We will use $C$ and $c$ to denote positive constants that depend at most on $d, \mu, \Omega$, and $Y_{s}$. Since the value of $\mu$ is not relevant in this study, we will assume $\mu=1$ in the rest of the paper for simplicity.

Acknowledgement. The author thanks Jinping Zhuge for several valuable comments. The author is also grateful for the valuable suggestions and corrections made by the anonymous referees. 


\section{Preliminaries}

Let $Y=[0,1]^{d}$ and $Y_{s}$ (solid part) be an open subset of $Y$ with Lipschitz boundary. We assume that $\operatorname{dist}\left(\partial Y, \partial Y_{s}\right)>0$ and that (the fluid part) $Y_{f}=Y \backslash \overline{Y_{s}}$ is connected. Let

$$
\omega=\bigcup_{z \in \mathbb{Z}^{d}}\left(Y_{f}+z\right)
$$

be the periodic repetition of $Y_{f}$. It is easy to see that the unbounded domain $\omega$ is connected, 1-periodic, and that $\partial \omega$ is locally Lipschitz.

For $1 \leq j \leq d$, let $\left(W_{j}(y), \pi_{j}(y)\right)=\left(W_{j}^{1}(y), \ldots, W_{j}^{d}(y), \pi_{j}(y)\right) \in H_{\mathrm{loc}}^{1}\left(\omega ; \mathbb{R}^{d}\right) \times L_{\mathrm{loc}}^{2}(\omega)$ be the 1-periodic solution of

$$
\left\{\begin{array}{rlrl}
-\Delta W_{j}+\nabla \pi_{j} & =e_{j} & & \text { in } \omega \\
\operatorname{div}\left(W_{j}\right)=0 & & \text { in } \omega \\
W_{j} & =0 & & \text { on } \partial \omega
\end{array}\right.
$$

with $\int_{Y_{f}} \pi_{j} d y=0$, where $e_{j}=(0, \ldots, 1, \ldots, 0)$ with 1 in the $j^{\text {th }}$ place. We extend $W_{j}$ to $\mathbb{R}^{d}$ by zero and define

$$
K_{j}^{i}=\int_{Y} W_{j}^{i}(y) d y
$$

Using

$$
K_{j}^{i}=\int_{Y} \nabla W_{j}^{\ell} \cdot \nabla W_{i}^{\ell} d y
$$

it is not hard to show that the $d \times d$ constant matrix $\left(K_{j}^{i}\right)$ is symmetric and positive definite.

Thanks to the assumption $\operatorname{dist}\left(\partial Y, \partial Y_{s}\right)>0$, we have $\partial \Omega_{\varepsilon}=\partial \Omega \cup \Gamma_{\varepsilon}$ and $\operatorname{dist}\left(\partial \Omega, \Gamma_{\varepsilon}\right) \geq c \varepsilon$, where

$$
\Gamma_{\varepsilon}=\Omega \cap \partial \Omega_{\varepsilon} \subset \partial(\varepsilon \omega) .
$$

For $f \in L^{2}\left(\Omega ; \mathbb{R}^{d}\right)$, let $\left(u_{\varepsilon}, p_{\varepsilon}\right)$ be a weak solution in $H_{0}^{1}\left(\Omega_{\varepsilon} ; \mathbb{R}^{d}\right) \times L^{2}\left(\Omega_{\varepsilon}\right)$ of the Dirichlet problem,

$$
\left\{\begin{aligned}
-\varepsilon^{2} \Delta u_{\varepsilon}+\nabla p_{\varepsilon}=f & \text { in } \Omega_{\varepsilon}, \\
\operatorname{div}\left(u_{\varepsilon}\right)=0 & \text { in } \Omega_{\varepsilon}, \\
u_{\varepsilon}=0 & \text { on } \partial \Omega_{\varepsilon},
\end{aligned}\right.
$$

with $\int_{\Omega_{\varepsilon}} p_{\varepsilon} d x=0$. We extend $u_{\varepsilon}$ to $\Omega$ by zero and still denote the extension by $u_{\varepsilon}$. Let $P_{\varepsilon}$ be the extension of $p_{\varepsilon}$, defined by

$$
P_{\varepsilon}(x)= \begin{cases}p_{\varepsilon}(x) & \text { if } x \in \Omega_{\varepsilon}, \\ f_{\varepsilon\left(Y_{f}+z_{k}\right)} p_{\varepsilon} & \text { if } x \in \varepsilon\left(Y_{s}+z_{k}\right) \text { and } \varepsilon\left(Y+z_{k}\right) \subset \Omega \text { for some } z_{k} \in \mathbb{Z}^{d}\end{cases}
$$

(see [15]).

Theorem 2.1. Let $\Omega$ be a bounded Lipschitz domain in $\mathbb{R}^{d}, d \geq 2$. Let $p_{0} \in H^{1}(\Omega)$ be the weak solution of the Neumann problem,

$$
\left\{\begin{aligned}
\frac{\partial}{\partial x_{i}} K_{j}^{i}\left(f_{j}-\frac{\partial p_{0}}{\partial x_{j}}\right) & =0 & & \text { in } \Omega \\
n_{i} K_{j}^{i}\left(f_{j}-\frac{\partial p_{0}}{\partial x_{j}}\right) & =0 & & \text { on } \partial \Omega
\end{aligned}\right.
$$


with $\int_{\Omega} p_{0} d x=0$, where $n=\left(n_{1}, \ldots, n_{d}\right)$ denotes the outward unit normal to $\partial \Omega$. Then, as $\varepsilon \rightarrow 0$,

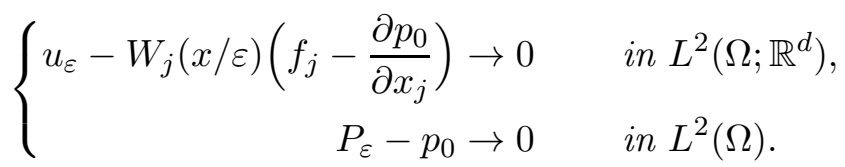

As indicated in Introduction, a proof of Theorem 2.1, using the method of two-scale convergence, may be found in [2, 3]. We do not use the theorem in this paper. However, we will need several other known results stated below.

Lemma 2.2. Let $\Omega$ be a bounded Lipschitz domain in $\mathbb{R}^{d}, d \geq 2$. Assume that $\Gamma_{\varepsilon} \neq \emptyset$. Let $u \in H^{1}\left(\Omega_{\varepsilon}\right)$ with $u=0$ on $\Gamma_{\varepsilon}$. Then

$$
\|u\|_{L^{2}\left(\Omega_{\varepsilon}\right)} \leq C \varepsilon\|\nabla u\|_{L^{2}\left(\Omega_{\varepsilon}\right)} .
$$

Proof. The case $u \in H_{0}^{1}\left(\Omega_{\varepsilon}\right)$ is more or less well known. See e.g. [5]. The proof for the case $u \in H^{1}\left(\Omega_{\varepsilon}\right)$ with $u_{\varepsilon}=0$ on $\Gamma_{\varepsilon}$ is the same. We sketch a proof here for the reader's convenience. Suppose $\varepsilon\left(Y+z_{k}\right) \subset \Omega$ for some $z_{k} \in \mathbb{Z}^{d}$. Since $u=0$ on $\Gamma_{\varepsilon}$, it follows by Poincaré's inequality that

$$
\int_{\varepsilon\left(Y_{f}+z_{k}\right)}|u|^{2} d x \leq C \varepsilon^{2} \int_{\varepsilon\left(Y_{f}+z_{k}\right)}|\nabla u|^{2} d x .
$$

Similarly,

$$
\int_{B\left(x_{0}, C \varepsilon\right) \cap \Omega_{\varepsilon}}|u|^{2} d x \leq C \varepsilon^{2} \int_{B\left(x_{0}, C \varepsilon\right) \cap \Omega_{\varepsilon}}|\nabla u|^{2} d x
$$

if $x_{0} \in \partial \Omega$ and $\varepsilon(Y+z) \subset B\left(x_{0}, C \varepsilon\right) \cap \Omega$ for some $z \in \mathbb{Z}^{d}$. The estimate (2.9) follows from (2.10) -(2.11) by a covering argument.

Lemma 2.3. Let $\Omega$ be a bounded Lipschitz domain in $\mathbb{R}^{d}, d \geq 2$. There exists a bounded linear operator

$$
R_{\varepsilon}: H^{1}\left(\Omega ; \mathbb{R}^{d}\right) \rightarrow H^{1}\left(\Omega_{\varepsilon}, \mathbb{R}^{d}\right)
$$

such that

$$
\left\{\begin{array}{l}
R_{\varepsilon}(u)=0 \quad \text { on } \Gamma_{\varepsilon} \quad \text { and } \quad R_{\varepsilon}(u)=u \quad \text { on } \partial \Omega \\
R_{\varepsilon}(u) \in H_{0}^{1}\left(\Omega_{\varepsilon} ; \mathbb{R}^{d}\right) \quad \text { if } u \in H_{0}^{1}\left(\Omega ; \mathbb{R}^{d}\right) \\
R_{\varepsilon}(u)=u \quad \text { in } \Omega_{\varepsilon} \quad \text { if } u=0 \text { on } \Gamma_{\varepsilon} \\
\operatorname{div}\left(R_{\varepsilon}(u)\right)=0 \quad \text { in } \Omega_{\varepsilon} \quad \text { if } \operatorname{div}(u)=0 \text { in } \Omega
\end{array}\right.
$$

and

$$
\varepsilon\left\|\nabla R_{\varepsilon}(u)\right\|_{L^{2}\left(\Omega_{\varepsilon}\right)}+\left\|R_{\varepsilon}(u)\right\|_{L^{2}\left(\Omega_{\varepsilon}\right)} \leq C\left\{\varepsilon\|\nabla u\|_{L^{2}(\Omega)}+\|u\|_{L^{2}(\Omega)}\right\},
$$

where $C$ depends only on $\Omega$ and $Y_{s}$. Moreover,

$$
\left\|\operatorname{div}\left(R_{\varepsilon}(u)\right)\right\|_{L^{2}\left(\Omega_{\varepsilon}\right)} \leq C\|\operatorname{div}(u)\|_{L^{2}(\Omega)}
$$

Proof. The proof is the similar to that of a lemma due to Tartar (in an appendix of [23], also see Lemma 1.7 in [4] $)$. Let $u \in H^{1}\left(\Omega ; \mathbb{R}^{d}\right)$. For each $\varepsilon(Y+z) \subset \Omega$, where $z \in \mathbb{Z}^{d}$, we define $R_{\varepsilon}(u)$ on $\varepsilon\left(Y_{f}+z\right)$ by the Dirichlet problem,

$$
\left\{\begin{aligned}
-\varepsilon^{2} \Delta R_{\varepsilon}(u)+\nabla q & =-\varepsilon^{2} \Delta u & & \text { in } \varepsilon\left(Y_{f}+z\right), \\
\operatorname{div}\left(R_{\varepsilon}(u)\right) & =\operatorname{div}(u)+\frac{1}{\left|\varepsilon\left(Y_{f}+z\right)\right|} \int_{\varepsilon\left(Y_{s}+z\right)} \operatorname{div}(u) d x & & \text { in } \varepsilon\left(Y_{f}+z\right), \\
R_{\varepsilon}(u) & =0 & & \text { on } \partial\left(\varepsilon\left(Y_{s}+z\right)\right), \\
R_{\varepsilon}(u) & =u & & \text { on } \partial(\varepsilon(Y+z)) .
\end{aligned}\right.
$$


If $x \in \Omega_{\varepsilon}$ and $x \notin \varepsilon\left(Y_{f}+z\right)$ for any $\varepsilon(Y+z) \subset \Omega$, we let $R_{\varepsilon}(u)(x)=u(x)$. It is not hard to show that $R_{\varepsilon}(u) \in H^{1}\left(\Omega_{\varepsilon} ; \mathbb{R}^{d}\right)$ satisfies the conditions in (2.13)-(2.15).

Lemma 2.4. Let $\Omega$ be a bounded Lipschitz domain in $\mathbb{R}^{d}, d \geq 2$. Suppose that $g \in L^{2}\left(\Omega_{\varepsilon}\right)$ and $\int_{\Omega_{\varepsilon}} g d x=0$. Then there exists $v_{\varepsilon} \in H_{0}^{1}\left(\Omega_{\varepsilon} ; \mathbb{R}^{d}\right)$ such that $\operatorname{div}\left(v_{\varepsilon}\right)=g$ in $\Omega_{\varepsilon}$ and

$$
\varepsilon\left\|\nabla v_{\varepsilon}\right\|_{L^{2}\left(\Omega_{\varepsilon}\right)}+\left\|v_{\varepsilon}\right\|_{L^{2}\left(\Omega_{\varepsilon}\right)} \leq C\|g\|_{L^{2}\left(\Omega_{\varepsilon}\right)}
$$

where $C$ depends only on $\Omega$ and $Y_{s}$.

Proof. See e.g. [5, pp.146-148].

We end this section with some observations on the rescaled solutions $\left(W_{j}(x / \varepsilon), \varepsilon \pi_{j}(x / \varepsilon)\right)$ in $\Omega_{\varepsilon}$. It follows from (2.2) by rescaling that

$$
\left\{\begin{aligned}
-\varepsilon^{2} \Delta\left\{W_{j}(x / \varepsilon)\right\}+\nabla\left\{\varepsilon \pi_{j}(x / \varepsilon)\right\} & =e_{j} & & \text { in } \varepsilon \omega, \\
\operatorname{div}\left(W_{j}(x / \varepsilon)\right) & =0 & & \text { in } \varepsilon \omega, \\
W_{j}(x / \varepsilon) & =0 & & \text { on } \partial(\varepsilon \omega) .
\end{aligned}\right.
$$

We extend both $W_{j}$ and $\pi_{j}$ to $\mathbb{R}^{d}$ by zero. Clearly,

$$
\left\{\begin{aligned}
\operatorname{div}\left(W_{j}(x / \varepsilon)\right) & =0 & & \text { in } \mathbb{R}^{d} \\
W_{j}(x / \varepsilon) & =0 & & \text { on } \Gamma_{\varepsilon}=\Omega \cap \partial \Omega_{\varepsilon} .
\end{aligned}\right.
$$

Note that in the construction of $\Omega_{\varepsilon}$, the holes near $\partial \Omega$ are not removed. As a result, the first equation in (2.18) needs to be modified for $\Omega_{\varepsilon}$. In fact, a computation using integration by parts shows that

$$
-\varepsilon^{2} \Delta\left\{W_{j}(x / \varepsilon)\right\}+\nabla\left\{\varepsilon \pi_{j}(x / \varepsilon)\right\}=e_{j}+\sigma_{\varepsilon, j} \quad \text { in } \Omega_{\varepsilon}
$$

where $\sigma_{\varepsilon, j} \in H^{-1}\left(\Omega_{\varepsilon} ; \mathbb{R}^{d}\right)$ is given by

$$
\begin{aligned}
& \left\langle\sigma_{\varepsilon, j}, \psi\right\rangle_{H^{-1}\left(\Omega_{\varepsilon}\right) \times H_{0}^{1}\left(\Omega_{\varepsilon}\right)} \\
& =\sum_{k}\left\{-\int_{\Omega \cap \varepsilon\left(Y_{s}+z_{k}\right)} e_{j} \cdot \psi d x-\varepsilon \int_{\bar{\Omega} \cap \partial\left(\varepsilon\left(Y_{s}+z_{k}\right)\right)}\left(\nabla W_{j}(x / \varepsilon) n-\pi_{j}(x / \varepsilon) n\right) \cdot \psi d \sigma\right\} .
\end{aligned}
$$

The sum in (2.21) is taken over those $k$ 's for which $z_{k} \in \mathbb{Z}^{d}$ and $\varepsilon\left(Y+z_{k}\right) \cap \partial \Omega \neq \emptyset$, and $n$ denotes the outward unit normal. Under the assumption that $\partial Y_{s}$ is $C^{1, \alpha}$, it is known that $\left|\nabla W_{j}\right|$ and $\pi_{j}$ are bounded in $\mathbb{R}^{d}$. It follows that if $g \in H_{\text {loc }}^{1}\left(\mathbb{R}^{d}\right)$ and $\psi \in H_{0}^{1}\left(\Omega_{\varepsilon} ; \mathbb{R}^{d}\right)$, then

$$
\left|\left\langle\sigma_{\varepsilon, j}, g \psi\right\rangle_{H^{-1}\left(\Omega_{\varepsilon}\right) \times H_{0}^{1}\left(\Omega_{\varepsilon}\right)}\right| \leq C \sum_{k}\left\{\int_{\varepsilon\left(Y+z_{k}\right)}|g \psi| d x+\varepsilon \int_{\partial\left(\varepsilon_{\left.\left(Y_{s}+z_{k}\right)\right)}\right.}|g \psi| d \sigma\right\}
$$

( $\psi$ is extended to $\mathbb{R}^{d}$ by zero). Using the inequality

$$
\int_{\partial\left(\varepsilon\left(Y_{s}+z_{k}\right)\right)}|u|^{2} d \sigma \leq C \varepsilon \int_{\varepsilon\left(Y+z_{k}\right)}|\nabla u|^{2} d x+C \varepsilon^{-1} \int_{\varepsilon\left(Y+z_{k}\right)}|u|^{2} d x,
$$

(2.9) and the Cauchy inequality, one may prove that

$$
\left|\left\langle\sigma_{\varepsilon, j}, g \psi\right\rangle_{H^{-1}\left(\Omega_{\varepsilon}\right) \times H_{0}^{1}\left(\Omega_{\varepsilon}\right)}\right| \leq C \varepsilon\left\{\varepsilon\|\nabla g\|_{L^{2}\left(\Sigma_{c \varepsilon}\right)}+\|g\|_{L^{2}\left(\Sigma_{c \varepsilon}\right)}\right\}\|\nabla \psi\|_{L^{2}\left(\Omega_{\varepsilon}\right)}
$$

for any $\psi \in H_{0}^{1}\left(\Omega_{\varepsilon} ; \mathbb{R}^{d}\right)$, where $\Sigma_{c \varepsilon}=\left\{x \in \mathbb{R}^{d}: \operatorname{dist}(x, \partial \Omega)<c \varepsilon\right\}$. 


\section{$3 \quad$ Energy estimates}

In this section we establish the energy estimates for the Dirichlet problem,

$$
\left\{\begin{aligned}
-\varepsilon^{2} \Delta u_{\varepsilon}+\nabla p_{\varepsilon} & =f+\varepsilon \operatorname{div}(F) & & \text { in } \Omega_{\varepsilon}, \\
\operatorname{div}\left(u_{\varepsilon}\right) & =g & & \text { in } \Omega_{\varepsilon}, \\
u_{\varepsilon} & =0 & & \text { on } \Gamma_{\varepsilon}, \\
u_{\varepsilon} & =h & & \text { on } \partial \Omega
\end{aligned}\right.
$$

where $(g, h)$ satisfies the compatibility condition,

$$
\int_{\Omega} g d x=\int_{\partial \Omega} h \cdot n d \sigma
$$

Theorem 3.1. Let $\Omega$ be a bounded domain in $\mathbb{R}^{d}, d \geq 2$ with Lipschitz boundary. Let $\left(u_{\varepsilon}, p_{\varepsilon}\right) \in$ $H^{1}\left(\Omega_{\varepsilon} ; \mathbb{R}^{d}\right) \times L^{2}\left(\Omega_{\varepsilon}\right)$ be a weak solution of (3.1) with $\int_{\Omega_{\varepsilon}} p_{\varepsilon} d x=0$. Then

$$
\begin{aligned}
& \varepsilon\left\|\nabla u_{\varepsilon}\right\|_{L^{2}\left(\Omega_{\varepsilon}\right)}+\left\|u_{\varepsilon}\right\|_{L^{2}\left(\Omega_{\varepsilon}\right)}+\left\|p_{\varepsilon}\right\|_{L^{2}\left(\Omega_{\varepsilon}\right)} \\
& \leq C\left\{\|f\|_{L^{2}\left(\Omega_{\varepsilon}\right)}+\|F\|_{L^{2}\left(\Omega_{\varepsilon}\right)}+\|g\|_{L^{2}\left(\Omega_{\varepsilon}\right)}+\|h\|_{L^{2}(\partial \Omega)}+\varepsilon\|h\|_{H^{1 / 2}(\partial \Omega)}\right\},
\end{aligned}
$$

for any $0<\varepsilon<1$, where $C$ depends only on $\Omega$ and $Y_{s}$.

Proof. We divide the proof into several steps.

Step 1. By Lemma 2.4, there exists $v_{\varepsilon} \in H_{0}^{1}\left(\Omega_{\varepsilon} ; \mathbb{R}^{d}\right)$ such that $\operatorname{div}\left(v_{\varepsilon}\right)=p_{\varepsilon}$ in $\Omega_{\varepsilon}$ and

$$
\varepsilon\left\|\nabla v_{\varepsilon}\right\|_{L^{2}\left(\Omega_{\varepsilon}\right)}+\left\|v_{\varepsilon}\right\|_{L^{2}\left(\Omega_{\varepsilon}\right)} \leq C\left\|p_{\varepsilon}\right\|_{L^{2}\left(\Omega_{\varepsilon}\right)} .
$$

By using $v_{\varepsilon}$ as a test function we see that

$$
\begin{aligned}
\left\|p_{\varepsilon}\right\|_{L^{2}\left(\Omega_{\varepsilon}\right)}^{2} & \leq \varepsilon^{2}\left\|\nabla u_{\varepsilon}\right\|_{L^{2}\left(\Omega_{\varepsilon}\right)}\left\|\nabla v_{\varepsilon}\right\|_{L^{2}\left(\Omega_{\varepsilon}\right)}+\|f\|_{L^{2}\left(\Omega_{\varepsilon}\right)}\left\|v_{\varepsilon}\right\|_{L^{2}\left(\Omega_{\varepsilon}\right)}+\varepsilon\|F\|_{L^{2}\left(\Omega_{\varepsilon}\right)}\left\|\nabla v_{\varepsilon}\right\|_{L^{2}\left(\Omega_{\varepsilon}\right)} \\
& \leq C\left\|p_{\varepsilon}\right\|_{L^{2}(\Omega)}\left\{\varepsilon\left\|\nabla u_{\varepsilon}\right\|_{L^{2}\left(\Omega_{\varepsilon}\right)}+\|f\|_{L^{2}\left(\Omega_{\varepsilon}\right)}+\|F\|_{L^{2}\left(\Omega_{\varepsilon}\right)}\right\},
\end{aligned}
$$

where we have used (3.4) for the last inequality. This gives

$$
\left\|p_{\varepsilon}\right\|_{L^{2}\left(\Omega_{\varepsilon}\right)} \leq C\left\{\varepsilon\left\|\nabla u_{\varepsilon}\right\|_{L^{2}\left(\Omega_{\varepsilon}\right)}+\|f\|_{L^{2}\left(\Omega_{\varepsilon}\right)}+\|F\|_{L^{2}\left(\Omega_{\varepsilon}\right)}\right\} \text {. }
$$

Step 2. We consider the case $h=0$ on $\partial \Omega$. This allows us to use the test function $u_{\varepsilon} \in H_{0}^{1}\left(\Omega_{\varepsilon} ; \mathbb{R}^{d}\right)$ to obtain

$$
\varepsilon^{2}\left\|\nabla u_{\varepsilon}\right\|_{L^{2}\left(\Omega_{\varepsilon}\right)}^{2} \leq\left\|p_{\varepsilon}\right\|_{L^{2}\left(\Omega_{\varepsilon}\right)}\|g\|_{L^{2}\left(\Omega_{\varepsilon}\right)}+\|f\|_{L^{2}\left(\Omega_{\varepsilon}\right)}\left\|u_{\varepsilon}\right\|_{L^{2}\left(\Omega_{\varepsilon}\right)}+\varepsilon\|F\|_{L^{2}\left(\Omega_{\varepsilon}\right)}\left\|\nabla u_{\varepsilon}\right\|_{L^{2}(\Omega)},
$$

where we have also used the Cauchy inequality. It follows from the inequality $\left\|u_{\varepsilon}\right\|_{L^{2}\left(\Omega_{\varepsilon}\right)} \leq$ $C \varepsilon\left\|\nabla u_{\varepsilon}\right\|_{L^{2}\left(\Omega_{\varepsilon}\right)}$ as well as the Cauchy inequality that

$$
\varepsilon^{2}\left\|\nabla u_{\varepsilon}\right\|_{L^{2}\left(\Omega_{\varepsilon}\right)}^{2} \leq C\left\{\left\|p_{\varepsilon}\right\|_{L^{2}\left(\Omega_{\varepsilon}\right)}\|g\|_{L^{2}\left(\Omega_{\varepsilon}\right)}+\|F\|_{L^{2}\left(\Omega_{\varepsilon}\right)}^{2}+\|f\|_{L^{2}\left(\Omega_{\varepsilon}\right)}^{2}\right\} .
$$

This, together with (3.5), yields (3.3) by the Cauchy inequality.

Step 3. In the general case, we let $(H, q) \in H^{1}\left(\Omega ; \mathbb{R}^{d}\right) \times L^{2}(\Omega)$ be a weak solution of

$$
-\Delta H+\nabla q=0 \quad \text { and } \quad \operatorname{div}(H)=\gamma \quad \text { in } \Omega,
$$


with boundary data $H=h$ on $\partial \Omega$, where

$$
\gamma=\frac{1}{|\Omega|} \int_{\partial \Omega} h \cdot n d \sigma
$$

Let $w_{\varepsilon}=R_{\varepsilon}(H)$, where $R_{\varepsilon}$ is the operator given by Lemma 2.3. Note that $w_{\varepsilon}=0$ on $\Gamma_{\varepsilon}, w_{\varepsilon}=h$ on $\partial \Omega$,

$$
\varepsilon\left\|\nabla w_{\varepsilon}\right\|_{L^{2}\left(\Omega_{\varepsilon}\right)}+\left\|w_{\varepsilon}\right\|_{L^{2}\left(\Omega_{\varepsilon}\right)} \leq C\left\{\varepsilon\|\nabla H\|_{L^{2}(\Omega)}+\|H\|_{L^{2}(\Omega)}\right\},
$$

and

$$
\left\|\operatorname{div}\left(w_{\varepsilon}\right)\right\|_{L^{2}\left(\Omega_{\varepsilon}\right)} \leq C|\gamma|
$$

Thus, $u_{\varepsilon}-w_{\varepsilon} \in H_{0}^{1}\left(\Omega_{\varepsilon} ; \mathbb{R}^{d}\right)$, and

$$
-\varepsilon^{2} \Delta\left(u_{\varepsilon}-w_{\varepsilon}\right)+\nabla p_{\varepsilon}=f+\varepsilon \operatorname{div}(F)+\varepsilon^{2} \Delta w_{\varepsilon} \quad \text { in } \Omega_{\varepsilon} .
$$

Hence, by Step 2, we obtain

$$
\begin{aligned}
& \varepsilon\left\|\nabla\left(u_{\varepsilon}-w_{\varepsilon}\right)\right\|_{L^{2}\left(\Omega_{\varepsilon}\right)}+\left\|u_{\varepsilon}-w_{\varepsilon}\right\|_{L^{2}\left(\Omega_{\varepsilon}\right)}+\left\|p_{\varepsilon}\right\|_{L^{2}\left(\Omega_{\varepsilon}\right)} \\
& \leq C\left\{\|f\|_{L^{2}\left(\Omega_{\varepsilon}\right)}+\|F\|_{L^{2}\left(\Omega_{\varepsilon}\right)}+\varepsilon\left\|\nabla w_{\varepsilon}\right\|_{L^{2}\left(\Omega_{\varepsilon}\right)}+\|g\|_{L^{2}\left(\Omega_{\varepsilon}\right)}+|\gamma|\right\},
\end{aligned}
$$

where we have used (3.8). It follows from (3.7) that

$$
\begin{aligned}
& \varepsilon\left\|\nabla u_{\varepsilon}\right\|_{L^{2}\left(\Omega_{\varepsilon}\right)}+\left\|u_{\varepsilon}\right\|_{L^{2}\left(\Omega_{\varepsilon}\right)}+\left\|p_{\varepsilon}\right\|_{L^{2}\left(\Omega_{\varepsilon}\right)} \\
& \leq C\left\{\|f\|_{L^{2}\left(\Omega_{\varepsilon}\right)}+\|F\|_{L^{2}\left(\Omega_{\varepsilon}\right)}+\|g\|_{L^{2}\left(\Omega_{\varepsilon}\right)}+|\gamma|+\varepsilon\|\nabla H\|_{L^{2}(\Omega)}+\|H\|_{L^{2}(\Omega)}\right\} .
\end{aligned}
$$

Step 4. To estimate $\|\nabla H\|_{L^{2}(\Omega)}$ and $\|H\|_{L^{2}(\Omega)}$, we let

$$
\widetilde{H}=H-\gamma d^{-1}\left(x-x_{0}\right),
$$

where $x_{0} \in \Omega$ is fixed. Note that

$$
-\Delta \widetilde{H}+\nabla q=0 \quad \text { and } \quad \operatorname{div}(\widetilde{H})=0 \quad \text { in } \Omega .
$$

By the energy estimates,

$$
\|\nabla \widetilde{H}\|_{L^{2}(\Omega)} \leq C\|\widetilde{H}\|_{H^{1 / 2}(\partial \Omega)} \leq C\|h\|_{H^{1 / 2}(\partial \Omega)},
$$

and by the nontangential-maximal-function estimates for the Stokes equations in [6],

$$
\|\widetilde{H}\|_{L^{2}(\Omega)} \leq C\|\widetilde{H}\|_{L^{2}(\partial \Omega)} \leq C\|h\|_{L^{2}(\partial \Omega)} .
$$

It follows that

$$
\varepsilon\|\nabla H\|_{L^{2}(\Omega)}+\|H\|_{L^{2}(\Omega)} \leq C\left\{\varepsilon\|h\|_{H^{1 / 2}(\partial \Omega)}+\|h\|_{L^{2}(\partial \Omega)}\right\} .
$$

This, together with (3.9), completes the proof.

Remark 3.2. If we replace the right-hand side $f+\varepsilon \operatorname{div}(F)$ of the first equation in (3.1) by some $\sigma_{\varepsilon} \in H^{-1}\left(\Omega_{\varepsilon} ; \mathbb{R}^{d}\right)$ that satisfies the condition

$$
\left|\left\langle\sigma_{\varepsilon}, \psi\right\rangle_{H^{-1}\left(\Omega_{\varepsilon}\right) \times H_{0}^{1}\left(\Omega_{\varepsilon}\right)}\right| \leq \varepsilon N\|\nabla \psi\|_{L^{2}\left(\Omega_{\varepsilon}\right)}
$$

for any $\psi \in H_{0}^{1}\left(\Omega_{\varepsilon} ; \mathbb{R}^{d}\right)$ and some $N=N\left(\sigma_{\varepsilon}\right)>0$, then the same argument as in the proof of Theorem 3.1 gives

$$
\varepsilon\left\|\nabla u_{\varepsilon}\right\|_{L^{2}\left(\Omega_{\varepsilon}\right)}+\left\|u_{\varepsilon}\right\|_{L^{2}\left(\Omega_{\varepsilon}\right)}+\left\|p_{\varepsilon}\right\|_{L^{2}\left(\Omega_{\varepsilon}\right)} \leq C\left\{N+\|g\|_{L^{2}\left(\Omega_{\varepsilon}\right)}+\|h\|_{L^{2}(\partial \Omega)}+\varepsilon\|h\|_{H^{1 / 2}(\partial \Omega)}\right\}
$$

for any $0<\varepsilon<1$, where $C$ depends only on $\Omega$ and $Y_{s}$. Note that if $\sigma_{\varepsilon}=f+\varepsilon \operatorname{div}(F)$, then $N=C\left\{\|f\|_{L^{2}\left(\Omega_{\varepsilon}\right)}+\|F\|_{L^{2}\left(\Omega_{\varepsilon}\right)}\right\}$. 


\section{Correctors for tangential boundary data}

Consider the Dirichlet problem,

$$
\left\{\begin{aligned}
-\varepsilon^{2} \Delta u_{\varepsilon}+\nabla p_{\varepsilon}=0 & \text { in } \Omega_{\varepsilon}, \\
\operatorname{div}\left(u_{\varepsilon}\right)=0 & \text { in } \Omega_{\varepsilon}, \\
u_{\varepsilon}=0 & \text { on } \Gamma_{\varepsilon} \\
u_{\varepsilon}=h & \text { on } \partial \Omega
\end{aligned}\right.
$$

with boundary data $h$ satisfying the condition

$$
h \cdot n=0 \quad \text { on } \partial \Omega .
$$

The goal of this section is to prove the following.

Theorem 4.1. Let $\Omega$ be a bounded Lipschitz domain in $\mathbb{R}^{d}, d \geq 2$. Let $\left(u_{\varepsilon}, p_{\varepsilon}\right)$ be a weak solution in $H^{1}\left(\Omega_{\varepsilon} ; \mathbb{R}^{d}\right) \times L^{2}\left(\Omega_{\varepsilon}\right)$ of (4.1) with $\int_{\Omega_{\varepsilon}} p_{\varepsilon} d x=0$, where $h \in H^{1}\left(\partial \Omega ; \mathbb{R}^{d}\right)$ satisfies (4.2). Then

$$
\varepsilon\left\|\nabla u_{\varepsilon}\right\|_{L^{2}\left(\Omega_{\varepsilon}\right)}+\left\|u_{\varepsilon}\right\|_{L^{2}\left(\Omega_{\varepsilon}\right)}+\left\|p_{\varepsilon}\right\|_{L^{2}\left(\Omega_{\varepsilon}\right)} \leq C \sqrt{\varepsilon}\left\{\|h\|_{L^{2}(\partial \Omega)}+\varepsilon\left\|\nabla_{\tan } h\right\|_{L^{2}(\partial \Omega)}\right\},
$$

where $\nabla_{\tan } h$ denotes the tangential gradient of $h$ on $\partial \Omega$.

Let

$$
\begin{aligned}
D_{r} & =\left\{\left(x^{\prime}, x_{d}\right) \in \mathbb{R}^{d}:\left|x^{\prime}\right|<r \quad \text { and } \quad \psi\left(x^{\prime}\right)<x_{d}<100 d(M+1) r\right\}, \\
I_{r} & =\left\{\left(x^{\prime}, \psi\left(x^{\prime}\right)\right) \in \mathbb{R}^{d}:\left|x^{\prime}\right|<r\right\},
\end{aligned}
$$

where $\psi: \mathbb{R}^{d-1} \rightarrow \mathbb{R}$ is a Lipschitz function such that $\psi(0)=0$ and $\|\nabla \psi\|_{\infty} \leq M$.

Lemma 4.2. Let $(v, q)$ be a weak solution in $H^{1}\left(D_{r} ; \mathbb{R}^{d}\right) \times L^{2}\left(D_{r}\right)$ of the Dirichlet problem,

$$
\left\{\begin{array}{rlrl}
-\Delta v+\nabla q=0 & & \text { in } D_{r}, \\
\operatorname{div}(v)=0 & & \text { in } D_{r}, \\
v & =g & & \text { on } \partial D_{r}
\end{array}\right.
$$

where $0<r<\infty$ and $g \in H^{1}\left(\partial D_{r} ; \mathbb{R}^{d}\right)$ satisfies the condition $\int_{\partial D_{r}} g \cdot n d \sigma=0$. Then

$$
\int_{\partial D_{r}}|\nabla v|^{2} d \sigma \leq C \int_{\partial D_{r}}\left|\nabla_{\tan } v\right|^{2} d \sigma
$$

where $C$ depends only on $d$ and $M$.

Proof. By dilation we may assume $r=1$, in which case the Rellich estimate (4.6) was proved in [6. Theorem 4.15].

Lemma 4.3. Let $(v, q)$ be a weak solution of (4.5) with $r=2$. Then

$$
\int_{I_{1}}|\nabla v|^{2} d \sigma \leq C \int_{I_{2}}\left|\nabla_{\tan } g\right|^{2} d \sigma+C \int_{D_{2}}|\nabla v|^{2} d x,
$$

where $C$ depends only on $d$ and $M$. 
Proof. It follows from (4.6) that for $1<r<2$,

$$
\int_{I_{1}}|\nabla v|^{2} d \sigma \leq C \int_{\partial D_{r}}|\nabla v|^{2} d \sigma \leq C \int_{\partial D_{r}}\left|\nabla_{\tan } v\right|^{2} d \sigma .
$$

Hence,

$$
\int_{I_{1}}|\nabla v|^{2} d \sigma \leq C \int_{I_{2}}\left|\nabla_{\tan } g\right|^{2} d \sigma+C \int_{D_{2} \cap \partial D_{r}}|\nabla v|^{2} d \sigma
$$

By integrating the inequality above in $r$ over the interval $(1,2)$, we obtain (4.7).

Proof of Theorem 4.1. We start with the observation,

$$
\varepsilon^{2} \int_{\Omega_{\varepsilon}}\left|\nabla u_{\varepsilon}\right|^{2} d x=\varepsilon^{2} \int_{\partial \Omega} \frac{\partial u_{\varepsilon}}{\partial n} \cdot u_{\varepsilon} d \sigma
$$

where we have used (4.1) and (4.2). It follows by the Cauchy inequality that

$$
\varepsilon^{2} \int_{\Omega_{\varepsilon}}\left|\nabla u_{\varepsilon}\right|^{2} d x \leq \varepsilon^{2}\left\|\nabla u_{\varepsilon}\right\|_{L^{2}(\partial \Omega)}\|h\|_{L^{2}(\partial \Omega)}
$$

We will show that

$$
\int_{\partial \Omega}\left|\nabla u_{\varepsilon}\right|^{2} d \sigma \leq C \int_{\partial \Omega}\left|\nabla_{\tan } h\right|^{2} d \sigma+\frac{C}{\varepsilon} \int_{\Sigma_{c \varepsilon}}\left|\nabla u_{\varepsilon}\right|^{2} d x
$$

where $\Sigma_{c \varepsilon}=\{x \in \Omega: \operatorname{dist}(x, \partial \Omega)<c \varepsilon\} \subset \Omega_{\varepsilon}$. Assume (4.9) for a moment. Then

$$
\left\|\nabla u_{\varepsilon}\right\|_{L^{2}(\partial \Omega)} \leq C\left\|\nabla_{\tan } h\right\|_{L^{2}(\partial \Omega)}+C \varepsilon^{-1 / 2}\left\|\nabla u_{\varepsilon}\right\|_{L^{2}\left(\Omega_{\varepsilon}\right)}
$$

This, together with (4.8) and the Cauchy inequality, gives

$$
\begin{aligned}
\varepsilon^{2}\left\|\nabla u_{\varepsilon}\right\|_{L^{2}\left(\Omega_{\varepsilon}\right)}^{2} & \leq C \varepsilon^{2}\|h\|_{L^{2}(\partial \Omega)}\left\|\nabla_{\tan } h\right\|_{L^{2}(\partial \Omega)}+C \varepsilon^{3 / 2}\|h\|_{L^{2}(\partial \Omega)}\left\|\nabla u_{\varepsilon}\right\|_{L^{2}\left(\Omega_{\varepsilon}\right)} \\
& \leq C \varepsilon^{2}\|h\|_{L^{2}(\partial \Omega)}\left\|\nabla_{\tan } h\right\|_{L^{2}(\partial \Omega)}+C \varepsilon\|h\|_{L^{2}(\partial \Omega)}^{2}+(1 / 2) \varepsilon^{2}\left\|\nabla u_{\varepsilon}\right\|_{L^{2}\left(\Omega_{e}\right)}^{2}
\end{aligned}
$$

which yields the estimate for $\varepsilon\left\|\nabla u_{\varepsilon}\right\|_{L^{2}\left(\Omega_{\varepsilon}\right)}$ in (4.3). The estimate for $\left\|u_{\varepsilon}\right\|_{L^{2}\left(\Omega_{\varepsilon}\right)}$ follows by (2.9)), while the bound for $\left\|p_{\varepsilon}\right\|_{L^{2}\left(\Omega_{\varepsilon}\right)}$ follows from (3.5).

It remains to prove (4.9). To this end, we shall prove in a few lines below that

$$
\int_{B\left(x_{0}, c_{0} \varepsilon\right) \cap \partial \Omega}\left|\nabla u_{\varepsilon}\right|^{2} d \sigma \leq C \int_{B\left(x_{0}, c_{1} \varepsilon\right) \cap \partial \Omega}\left|\nabla_{\tan } h\right|^{2} d \sigma+\frac{C}{\varepsilon} \int_{B\left(x_{0}, c_{1} \varepsilon\right) \cap \Omega}\left|\nabla u_{\varepsilon}\right|^{2} d x
$$

for any $x_{0} \in \partial \Omega$, where $0<c_{0}<c_{1}$ are sufficiently small. The desired estimate (4.9) follows from (4.10) by covering $\partial \Omega$ with a finite number of balls $\left\{B\left(x_{k}, c_{0} \varepsilon\right)\right\}$ centered on $\partial \Omega$.

Finally, we note that if $v(x)=u_{\varepsilon}(\varepsilon x)$ and $q(x)=\varepsilon^{-1} p_{\varepsilon}(\varepsilon x)$, then $-\Delta v+\nabla q=0$ and $\operatorname{div}(v)=0$. As a result, the estimate (4.10) follows from (4.7) by a translation and rotation of the coordinate system. We point out that since the constant $C$ in (4.7) depends only on $d$ and $M$, the constant $C$ in (4.10) depends only on $d$ and the Lipschitz character of $\Omega$. In particular, $C$ does not depend on $\varepsilon$.

As a corollary of Theorem 4.1, we are able to construct a tangential boundary layer corrector. 
Theorem 4.4. Let $\Omega$ be bounded domain with $C^{2, \alpha}$ boundary for some $\alpha>0$. Also assume that $\partial Y_{s}$ is $C^{1, \alpha}$. Let $\left(\Psi_{t}, q_{t}\right)$ be a weak solution in $H^{1}\left(\Omega_{\varepsilon} ; \mathbb{R}^{d}\right) \times L^{2}\left(\Omega_{\varepsilon}\right)$ of the Dirichlet problem (4.1) with $\int_{\Omega_{\varepsilon}} q_{t} d x=0$, where the boundary data $h$ is given by

$$
h=b-W_{j}(x / \varepsilon)\left(f_{j}-\frac{\partial p_{0}}{\partial x_{j}}\right)+\left[-b \cdot n+n_{i} W_{j}^{i}(x / \varepsilon)\left(f_{j}-\frac{\partial p_{0}}{\partial x_{j}}\right)\right] n,
$$

$f \in C^{1,1 / 2}\left(\bar{\Omega} ; \mathbb{R}^{d}\right), b \in H^{1}\left(\partial \Omega ; \mathbb{R}^{d}\right)$ satisfies $\int_{\partial \Omega} b \cdot n d \sigma=0$, and $p_{0}$ is the solution of the Neumann problem,

$$
\left\{\begin{aligned}
K_{j}^{i} \frac{\partial}{\partial x_{i}}\left(f_{j}-\frac{\partial p_{0}}{\partial x_{j}}\right) & =0 & & \text { in } \Omega, \\
n_{i} K_{j}^{i}\left(f_{j}-\frac{\partial p_{0}}{\partial x_{j}}\right) & =b \cdot n & & \text { on } \partial \Omega,
\end{aligned}\right.
$$

with $\int_{\Omega} p_{0} d x=0$. Then

$$
\begin{aligned}
& \varepsilon\left\|\nabla \Psi_{t}\right\|_{L^{2}\left(\Omega_{\varepsilon}\right)}+\left\|\Psi_{t}\right\|_{L^{2}\left(\Omega_{\varepsilon}\right)}+\left\|q_{t}\right\|_{L^{2}\left(\Omega_{\varepsilon}\right)} \\
& \leq C \sqrt{\varepsilon}\left\{\left\|f-\nabla p_{0}\right\|_{L^{2}(\partial \Omega)}+\|b\|_{L^{2}(\partial \Omega)}+\varepsilon\left\|\nabla_{\tan }\left(f-\nabla p_{0}\right)\right\|_{L^{2}(\partial \Omega)}+\varepsilon\left\|\nabla_{\tan } b\right\|_{L^{2}(\partial \Omega)}\right\}
\end{aligned}
$$

for any $0<\varepsilon<1$.

Proof. Note that $h \cdot n=0$ on $\partial \Omega$. Also, under the assumption that $\partial Y_{s}$ is $C^{1, \alpha}$, we have $W_{j}=$ $W_{j}(y) \in C^{1}(\bar{\omega})$. It follows that

$$
\|h\|_{L^{2}(\partial \Omega)} \leq C\left\{\left\|f-\nabla p_{0}\right\|_{L^{2}(\partial \Omega)}+\|b\|_{L^{2}(\partial \Omega)}\right\}
$$

and

$$
\begin{aligned}
& \left\|\nabla_{\tan } h\right\|_{L^{2}(\partial \Omega)} \\
& \leq C\left\{\varepsilon^{-1}\left\|f-\nabla p_{0}\right\|_{L^{2}(\partial \Omega)}+\left\|\nabla_{\tan }\left(f-\nabla p_{0}\right)\right\|_{L^{2}(\partial \Omega)}+\left\|\nabla_{\tan } b\right\|_{L^{2}(\partial \Omega)}+\|b\|_{L^{2}(\partial \Omega)}\right\} .
\end{aligned}
$$

As a result, the estimate (4.13) follows readily from (4.3).

\section{Correctors for normal boundary data}

In this section we consider the Dirichlet problem (4.1), where the boundary data $h$ is given by

$$
h=\left\{n_{i}\left[W_{j}^{i}(x / \varepsilon)-K_{j}^{i}\right] g_{j}-\gamma\right\} n,
$$

where $g=\left(g_{1}, g_{2}, \ldots, g_{d}\right) \in H^{1}\left(\partial \Omega ; \mathbb{R}^{d}\right)$, and $\gamma \in \mathbb{R}$ is chosen so that $\int_{\partial \Omega} h \cdot n d \sigma=0$, i.e.,

$$
\gamma=\frac{1}{|\partial \Omega|} \int_{\partial \Omega} n_{i}\left[W_{j}^{i}(x / \varepsilon)-K_{j}^{i}\right] g_{j} d \sigma .
$$

The goal of this section is to prove the following.

Theorem 5.1. Let $\Omega$ be a bounded $C^{2, \alpha}$ domain in $\mathbb{R}^{d}$ for some $\alpha>0$. Also assume that $\partial Y_{s}$ is $C^{1, \alpha}$. Let $\left(u_{\varepsilon}, p_{\varepsilon}\right)$ be a weak solution in $H^{1}\left(\Omega_{\varepsilon} ; \mathbb{R}^{d}\right) \times L^{2}\left(\Omega_{\varepsilon}\right)$ of (4.1) with $\int_{\Omega_{\varepsilon}} p_{\varepsilon} d x=0$, where $h$ is given by (5.1). Then

$$
\varepsilon\left\|\nabla u_{\varepsilon}\right\|_{L^{2}\left(\Omega_{\varepsilon}\right)}+\left\|u_{\varepsilon}\right\|_{L^{2}\left(\Omega_{\varepsilon}\right)}+\left\|p_{\varepsilon}\right\|_{L^{2}\left(\Omega_{\varepsilon}\right)} \leq C \sqrt{\varepsilon}\left\{\|g\|_{L^{2}(\partial \Omega)}+\sqrt{\varepsilon}\left\|\nabla_{\tan } g\right\|_{L^{2}(\partial \Omega)}\right\},
$$

for any $0<\varepsilon<1$. 
We will prove a series of lemmas before we give the proof of Theorem 5.1. We begin with an estimate for $\|h\|_{H^{1 / 2}(\partial \Omega)}$.

Lemma 5.2. Let $h$ be given by (5.1). Then

$$
\|h\|_{H^{1 / 2}(\partial \Omega)} \leq C \varepsilon^{-1 / 2}\left\{\|g\|_{L^{2}(\partial \Omega)}+\varepsilon\left\|\nabla_{\tan } g\right\|_{L^{2}(\partial \Omega)}\right\}
$$

for any $0<\varepsilon<1$.

Proof. Note that

$$
\begin{aligned}
\|h\|_{H^{1 / 2}(\partial \Omega)} & \leq C\|h\|_{L^{2}(\partial \Omega)}^{1 / 2}\|h\|_{H^{1}(\partial \Omega)}^{1 / 2} \\
& \leq C\left\{\varepsilon^{-1 / 2}\|h\|_{L^{2}(\partial \Omega)}+\varepsilon^{1 / 2}\left\|\nabla_{\tan } h\right\|_{L^{2}(\partial \Omega)}\right\}
\end{aligned}
$$

where we have used the Cauchy inequality. It is easy to see that $\|h\|_{L^{2}(\partial \Omega)} \leq C\|g\|_{L^{2}(\partial \Omega)}$, and

$$
\left\|\nabla_{\tan } h\right\|_{L^{2}(\partial \Omega)} \leq C\left\{\varepsilon^{-1}\|g\|_{L^{2}(\partial \Omega)}+\left\|\nabla_{\tan } g\right\|_{L^{2}(\partial \Omega)}\right\},
$$

where we have used the fact $W_{j}=W_{j}(y) \in C^{1}\left(\bar{\omega} ; \mathbb{R}^{d}\right)$. It follows that

$$
\|h\|_{H^{1 / 2}(\partial \Omega)} \leq C \varepsilon^{-1 / 2}\left\{\|g\|_{L^{2}(\partial \Omega)}+\varepsilon\left\|\nabla_{\tan } g\right\|_{L^{2}(\partial \Omega)}\right\}
$$

for any $0<\varepsilon<1$.

Lemma 5.3. There exist 1-periodic functions $\phi_{i j}^{\ell} \in H_{l o c}^{1}\left(\mathbb{R}^{d}\right)$, where $1 \leq i, j, \ell \leq d$, such that

$$
\frac{\partial \phi_{i j}^{\ell}}{\partial y_{i}}=W_{j}^{\ell}(y)-K_{j}^{\ell} \quad \text { and } \quad \phi_{i j}^{\ell}=-\phi_{\ell j}^{i}
$$

where the index $i$ is summed from 1 to $d$.

Proof. The proof is the same as Lemma 3.1 in [13. Since

$$
\int_{Y}\left(W_{j}^{\ell}(y)-K_{j}^{\ell}\right) d y=0
$$

one may solve the periodic boundary value problem,

$$
\left\{\begin{array}{l}
\Delta f_{j}^{\ell}=W_{j}^{\ell}-K_{j}^{\ell} \quad \text { in } Y, \\
f_{j}^{\ell} \text { is 1-periodic. }
\end{array}\right.
$$

Let

$$
\phi_{i j}^{\ell}=\frac{\partial f_{j}^{\ell}}{\partial y_{i}}-\frac{\partial f_{j}^{i}}{\partial y_{\ell}} .
$$

Then $\phi_{i j}^{\ell}=-\phi_{\ell j}^{i}$. Using

$$
\frac{\partial}{\partial y_{\ell}} W_{j}^{\ell}=0
$$

we obtain the first equation in (5.5). 
Remark 5.4. Using (5.5), for $1 \leq j \leq d$, we may write

$$
\begin{aligned}
n_{\ell}\left[W_{j}^{\ell}(x / \varepsilon)-K_{j}^{\ell}\right] & =\varepsilon n_{\ell} \frac{\partial}{\partial x_{i}}\left\{\phi_{i j}^{\ell}(x / \varepsilon)\right\} \\
& =\frac{\varepsilon}{2}\left\{n_{\ell} \frac{\partial}{\partial x_{i}}-n_{i} \frac{\partial}{\partial x_{\ell}}\right\}\left\{\phi_{i j}^{\ell}(x / \varepsilon)\right\},
\end{aligned}
$$

where the skew-symmetric property is used for the last step. It follows from an integration by parts on $\partial \Omega$ that

$$
\int_{\partial \Omega} n_{\ell}\left[W_{j}^{\ell}(x / \varepsilon)-K_{j}^{\ell}\right] \psi d \sigma(x)=-\frac{\varepsilon}{2} \int_{\partial \Omega} \phi_{i j}^{\ell}(x / \varepsilon)\left\{n_{\ell} \frac{\partial}{\partial x_{i}}-n_{i} \frac{\partial}{\partial x_{\ell}}\right\} \psi d \sigma(x) .
$$

This, in particular, implies that

$$
|\gamma| \leq C \varepsilon\left\|\nabla_{\tan } g\right\|_{L^{2}(\partial \Omega)},
$$

where $\gamma$ is given by (15.2), assuming that $\left(\phi_{i j}^{\ell}\right)$ are bounded.

Let

$$
\Sigma_{\rho}=\{x \in \Omega: \operatorname{dist}(x, \partial \Omega)<\rho\} .
$$

Lemma 5.5. Let $T$ be the operator defined by

$$
T(f)(x)=\int_{\partial \Omega} \frac{f(y)}{|x-y|^{d}} d \sigma(y) .
$$

Then

for any $0<\varepsilon<1$, and

$$
\int_{\Omega \backslash \Sigma_{\varepsilon}}|T(f)|^{2} d x \leq C \varepsilon^{-1} \int_{\partial \Omega}|f|^{2} d \sigma
$$

for any $\delta>1$.

$$
\int_{\Omega}[\operatorname{dist}(x, \partial \Omega)]^{\delta}|T(f)|^{2} d x \leq C_{\delta} \int_{\partial \Omega}|f|^{2} d \sigma
$$

Proof. It is not hard to see that

$$
\int_{\partial \Omega} \frac{d \sigma(y)}{|x-y|^{d}} \leq \frac{C}{\operatorname{dist}(x, \partial \Omega)}
$$

for any $x \in \Omega$. By the Cauchy inequality,

$$
|T(f)(x)|^{2} \leq \frac{C}{\operatorname{dist}(x, \partial \Omega)} \int_{\partial \Omega} \frac{|f(y)|^{2}}{|x-y|^{d}} d \sigma(y) .
$$

The estimate (5.10) follows by integrating the inequality above and using Fubini's Theorem. A similar argument gives 5.11 .

Lemma 5.6. Let $(H, q)$ be a weak solution in $H^{1}\left(\Omega ; \mathbb{R}^{d}\right) \times L^{2}(\Omega)$ of the Dirichlet problem,

$$
\left\{\begin{aligned}
-\Delta H+\nabla q=0 & \text { in } \Omega, \\
\operatorname{div}(H)=0 & \text { in } \Omega, \\
H=h & \text { on } \partial \Omega,
\end{aligned}\right.
$$

where $h$ is given by (5.1). Then

$$
\varepsilon\|\nabla H\|_{L^{2}(\Omega)}+\|H\|_{L^{2}(\Omega)} \leq C \sqrt{\varepsilon}\left\{\|g\|_{L^{2}(\partial \Omega)}+\sqrt{\varepsilon}\left\|\nabla_{\tan } g\right\|_{L^{2}(\partial \Omega)}\right\},
$$

for any $0<\varepsilon<1$. 
Proof. We first point out that by the standard energy estimates for the Stokes equations,

$$
\|\nabla H\|_{L^{2}(\Omega)} \leq C\|h\|_{H^{1 / 2}(\partial \Omega)} .
$$

In view of (5.4), this gives

$$
\varepsilon\|\nabla H\|_{L^{2}(\Omega)} \leq C \sqrt{\varepsilon}\left\{\|g\|_{L^{2}(\partial \Omega)}+\varepsilon\left\|\nabla_{\tan } g\right\|_{L^{2}(\partial \Omega)}\right\} .
$$

Next, we use the nontangential-maximal-function estimate,

$$
\left\|(H)^{*}\right\|_{L^{2}(\partial \Omega)} \leq C\|h\|_{L^{2}(\partial \Omega)},
$$

to bound $H$ on $\Sigma_{\varepsilon}$. The estimate (5.14) was proved in [6] for a Lipschitz domain $\Omega$, where the nontangential maximal function $(H)^{*}$ is defined by

$$
(H)^{*}(x)=\sup \left\{|H(y)|: y \in \Omega \text { and }|y-x|<C_{0} \operatorname{dist}(y, \partial \Omega)\right\}
$$

for $x \in \partial \Omega$. It follows that

$$
\begin{aligned}
\|H\|_{L^{2}\left(\Sigma_{\varepsilon}\right)} & \leq C \varepsilon^{1 / 2}\left\|(H)^{*}\right\|_{L^{2}(\partial \Omega)} \leq C \varepsilon^{1 / 2}\|h\|_{L^{2}(\partial \Omega)} \\
& \leq C \varepsilon^{1 / 2}\|g\|_{L^{2}(\partial \Omega)} .
\end{aligned}
$$

It remains to bound $H$ on $\Omega \backslash \Sigma_{\varepsilon}$. To this end, we let $(G(x, y), \Pi(x, y))$ denote the matrix of Green functions for the Stokes equation (5.12) in $\Omega$. That is, for each fixed $x \in \Omega, G(x, y)=$ $\left(G^{i j}(x, y)\right) \in H_{\text {loc }}^{2}\left(\Omega \backslash\{x\} ; \mathbb{R}^{d \times d}\right)$ and $\Pi(x, y)=\left(\Pi^{i}(x, y)\right) \in L_{\text {loc }}^{2}\left(\Omega \backslash\{x\} ; \mathbb{R}^{d}\right)$ satisfy

$$
\left\{\begin{aligned}
-\Delta_{y} G^{i j}(x, y)+\frac{\partial}{\partial y_{j}} \Pi^{i}(x, y) & =\delta_{x} \delta_{i j} & & \text { in } \Omega \backslash\{x\}, \\
\frac{\partial}{\partial y_{j}}\left(G^{i j}(x, y)\right) & =0 & & \text { in } \Omega \backslash\{x\}, \\
G^{i j}(x, y) & =0 & & \text { for } y \in \partial \Omega,
\end{aligned}\right.
$$

in the sense of distribution. We also require that

$$
\Pi(x, \cdot) \in L^{1}\left(\Omega ; \mathbb{R}^{d}\right) \quad \text { and } \quad \int_{\Omega} \Pi(x, y) d y=0 .
$$

Under the assumption that $\Omega$ is a bounded $C^{2, \alpha}$ domain for some $\alpha>0$, solutions of the Stokes equations (5.12) satisfy the $C^{1,1}$ estimate for $H$ and $C^{0,1}$ estimate for $q$, up to the boundary. It follows that

$$
\begin{aligned}
\left|\nabla_{x} G(x, y)\right|+\left|\nabla_{y} G(x, y)\right| & \leq C|x-y|^{1-d}, \\
\left|\nabla_{y} G(x, y)\right| & \leq C \operatorname{dist}(x, \partial \Omega)|x-y|^{-d}, \\
\left|\nabla_{x}^{2} G(x, y)\right|+\left|\nabla_{y}^{2} G(x, y)\right|+\left|\nabla_{x} \nabla_{y} G(x, y)\right| & \leq C|x-y|^{-d},
\end{aligned}
$$

and that

$$
\begin{aligned}
|\Pi(x, y)| & \leq C|x-y|^{1-d}, \\
|\Pi(x, y)-\Pi(x, z)| & \leq \operatorname{dist}(x, \partial \Omega)\left\{|x-y|^{-d}+|x-z|^{-d}\right\}, \\
\left|\nabla_{y} \Pi(x, y)\right| & \leq C|x-y|^{-d},
\end{aligned}
$$


for any $x, y \in \Omega$ and $x \neq y, x \neq z$. See e.g. [7, 21]. This allows us to represent the solution $H(x)$ by

$$
H^{i}(x)=-\int_{\partial \Omega}\left\{n_{k}(y) \frac{\partial}{\partial y_{k}} G^{i j}(x, y)-\left[\Pi^{i}(x, y)-\Pi^{i}(x, z)\right] n_{j}(y)\right\} h^{j}(y) d \sigma(y)
$$

for any $x \in \Omega$, where $z \in \Omega$ and $z \neq x$ (due to the compatibility condition for $h$, the choice of $z$ is arbitrary). Using (5.6), we may write $h=h^{(1)}+h^{(2)}$, where

$$
\begin{aligned}
h^{(1), k} & =\frac{\varepsilon}{2}\left(n_{\ell} \frac{\partial}{\partial x_{i}}-n_{i} \frac{\partial}{\partial x_{\ell}}\right)\left\{\phi_{i j}^{\ell}(x / \varepsilon) g_{j} n_{k}\right\}, \\
h^{(2), k} & =-\frac{\varepsilon}{2} \phi_{i j}^{\ell}(x / \varepsilon)\left(n_{\ell} \frac{\partial}{\partial x_{i}}-n_{i} \frac{\partial}{\partial x_{\ell}}\right)\left(g_{j} n_{k}\right)-\gamma n_{k},
\end{aligned}
$$

for $1 \leq k \leq d$. Let $H^{(1)}(x), H^{(2)}(x)$ be given by (5.19), with $h$ being replaced by $h^{(1)}, h^{(2)}$, respectively. Observe that by the divergence theorem,

$$
\int_{\partial \Omega}\left(n_{\ell} \frac{\partial}{\partial x_{i}}-n_{i} \frac{\partial}{\partial x_{\ell}}\right) v \cdot w d \sigma=-\int_{\partial \Omega} v \cdot\left(n_{\ell} \frac{\partial}{\partial x_{i}}-n_{i} \frac{\partial}{\partial x_{\ell}}\right) w d \sigma
$$

for $1 \leq i, \ell \leq d$. It follows that

$$
\begin{aligned}
& \left|H^{(1)}(x)\right| \\
& \leq C \varepsilon \int_{\partial \Omega}\left\{\left|\nabla_{y} G(x, y)\right|+\left|\nabla_{y}^{2} G(x, y)\right|+\left|\nabla_{y} \Pi(x, y)\right|+|\Pi(x, y)-\Pi(x, z)|\right\}|g(y)| d \sigma(y) \\
& \leq C \varepsilon \int_{\partial \Omega} \frac{|g(y)|}{|x-y|^{d}} d \sigma(y),
\end{aligned}
$$

where we have used the estimates in (5.17) and (5.18). In view of Lemma 5.5, we obtain

$$
\left\|H^{(1)}\right\|_{L^{2}\left(\Omega \backslash \Sigma_{\varepsilon}\right)} \leq C \varepsilon^{1 / 2}\|g\|_{L^{2}(\partial \Omega)} .
$$

Finally, note that

$$
\begin{aligned}
\left|H^{(2)}(x)\right| & \leq C \varepsilon\left\|\nabla_{\tan } g\right\|_{L^{2}(\partial \Omega)} \\
& +C \varepsilon \int_{\partial \Omega}\left\{\left|\nabla_{y} G(x, y)\right|+|\Pi(x, y)-\Pi(x, z)|\right\}\left(|g(y)|+\left|\nabla_{\tan } g(y)\right|\right) d \sigma(y),
\end{aligned}
$$

where we have used (5.8). Using estimates in (5.17) and (5.18), we may deduce from (5.11) that

$$
\left\|H^{(2)}\right\|_{L^{2}(\Omega)} \leq C \varepsilon\left\{\left\|\nabla_{\tan } g\right\|_{L^{2}(\partial \Omega)}+\|g\|_{L^{2}(\partial \Omega)}\right\},
$$

which completes the proof.

We are now ready to give the proof of Theorem 5.1

Proof of Theorem [5.1. Let $\left(u_{\varepsilon}, p_{\varepsilon}\right)$ be a weak solution in $H^{1}\left(\Omega_{\varepsilon} ; \mathbb{R}^{d}\right) \times L^{2}\left(\Omega_{\varepsilon}\right)$ of (4.1) with $\int_{\Omega_{\varepsilon}} p_{\varepsilon} d x=$ 0 , where $h$ is given by (5.1). Let $(H, q)$ be a solution of (5.12) with boundary data $h$. It follows from (3.9) that

$$
\begin{aligned}
\varepsilon\left\|\nabla u_{\varepsilon}\right\|_{L^{2}\left(\Omega_{\varepsilon}\right)}+\left\|u_{\varepsilon}\right\|_{L^{2}\left(\Omega_{\varepsilon}\right)}+\left\|p_{\varepsilon}\right\|_{L^{2}\left(\Omega_{\varepsilon}\right)} & \leq C\left\{\varepsilon\|\nabla H\|_{L^{2}(\Omega)}+\|H\|_{L^{2}(\Omega)}\right\} \\
& \leq C \sqrt{\varepsilon}\left\{\|g\|_{L^{2}(\partial \Omega)}+\sqrt{\varepsilon}\left\|\nabla_{\tan } g\right\|_{L^{2}(\partial \Omega)}\right\}
\end{aligned}
$$

where we have used (5.13) for the last inequality. 
As a corollary of Theorem [5.1, we construct a normal boundary layer corrector.

Theorem 5.7. Let $\Omega$ be a bounded $C^{2, \alpha}$ domain for some $\alpha>0$. Also assume that $\partial Y_{s}$ is $C^{1, \alpha}$. Let $\left(\Psi_{n}, q_{n}\right)$ be a weak solution of (4.1) with $\int_{\Omega_{\varepsilon}} q_{n} d x=0$, where the boundary data $h$ is given by

$$
h=\left\{-n_{i} W_{j}^{i}(x / \varepsilon)\left(f_{j}-\frac{\partial p_{0}}{\partial x_{j}}\right)+b \cdot n-\gamma\right\} n
$$

$p_{0}$ is defined by (4.12), $b$ is the same as in Theorem 4.4, and $\gamma \in \mathbb{R}$ is such that $\int_{\partial \Omega} h \cdot n d \sigma=0$. Then

$$
\begin{aligned}
& \varepsilon\left\|\nabla \Psi_{n}\right\|_{L^{2}\left(\Omega_{\varepsilon}\right)}+\left\|\Psi_{n}\right\|_{L^{2}\left(\Omega_{\varepsilon}\right)}+\left\|q_{n}\right\|_{L^{2}\left(\Omega_{\varepsilon}\right)} \\
& \leq C \sqrt{\varepsilon}\left\{\left\|f-\nabla p_{0}\right\|_{L^{2}(\partial \Omega)}+\sqrt{\varepsilon}\left\|\nabla_{\tan }\left(f-\nabla p_{0}\right)\right\|_{L^{2}(\partial \Omega)}\right\}
\end{aligned}
$$

for any $0<\varepsilon<1$. Moreover,

$$
|\gamma| \leq C \varepsilon\left\|\nabla_{\tan }\left(f-\nabla p_{0}\right)\right\|_{L^{2}(\partial \Omega)} .
$$

Proof. Note that by the boundary condition in (4.12),

$$
h=\left\{-n_{\ell}\left[W_{j}^{\ell}(x / \varepsilon)-K_{j}^{\ell}\right]\left(f_{j}-\frac{\partial p_{0}}{\partial x_{j}}\right)-\gamma\right\} n \quad \text { on } \partial \Omega .
$$

As a result, the estimate (5.24) follows readily from Theorem 5.1 with $g=-\left(f-\nabla p_{0}\right)$.

\section{Convergence rates}

In this section we prove the following theorem, which contains Theorem 1.1 .

Theorem 6.1. Let $\Omega$ be a bounded $C^{2, \alpha}$ domain in $\mathbb{R}^{d}, d \geq 2$ for some $\alpha>0$. Also assume that $\partial Y_{s}$ is $C^{1, \alpha}$. Let $\left(u_{\varepsilon}, p_{\varepsilon}\right) \in H^{1}\left(\Omega_{\varepsilon} ; \mathbb{R}^{d}\right) \times L^{2}\left(\Omega_{\varepsilon} ; \mathbb{R}^{d}\right)$ be a weak solution of the Dirichlet problem,

$$
\left\{\begin{aligned}
-\varepsilon^{2} \Delta u_{\varepsilon}+\nabla p_{\varepsilon}=f & \text { in } \Omega_{\varepsilon}, \\
\operatorname{div}\left(u_{\varepsilon}\right)=0 & \text { in } \Omega_{\varepsilon}, \\
u_{\varepsilon}=0 & \text { on } \Gamma_{\varepsilon}, \\
u_{\varepsilon}=b & \text { on } \partial \Omega,
\end{aligned}\right.
$$

where $f \in C^{1,1 / 2}\left(\bar{\Omega} ; \mathbb{R}^{d}\right)$ and $b \in H^{1}\left(\partial \Omega ; \mathbb{R}^{d}\right)$ satisfies the compatibility condition $\int_{\partial \Omega} b \cdot n d \sigma=0$. Assume that $\int_{\Omega_{\varepsilon}} p_{\varepsilon} d x=0$. Then for $0<\varepsilon<1$,

$$
\begin{aligned}
& \left\|u_{\varepsilon}-W(x / \varepsilon)\left(f-\nabla p_{0}\right)\right\|_{L^{2}(\Omega)}+\left\|P_{\varepsilon}-p_{0}\right\|_{L^{2}(\Omega)} \\
& \quad+\left\|\varepsilon \nabla u_{\varepsilon}-\nabla W(x / \varepsilon)\left(f-\nabla p_{0}\right)\right\|_{L^{2}(\Omega)} \\
& \quad \leq C \sqrt{\varepsilon}\left\{\|f\|_{C^{1,1 / 2}(\Omega)}+\|b \cdot n\|_{H^{1}(\partial \Omega)}+\|b\|_{L^{2}(\partial \Omega)}+\varepsilon\left\|\nabla_{\tan } b\right\|_{L^{2}(\partial \Omega)}\right\}
\end{aligned}
$$

where $p_{0}$ is defined by (4.12), $P_{\varepsilon}$ is given by (2.6), and $C$ depends only on $\Omega$ and $Y_{s}$.

We begin by introducing a corrector for the divergence operator. For $1 \leq i, k \leq d$, let $\left(\chi_{i k}^{1}(y), \ldots, \chi_{i k}^{d}(y), \pi_{2, i k}(y)\right) \in H_{\mathrm{loc}}^{1}\left(\omega ; \mathbb{R}^{d}\right) \times L_{\mathrm{loc}}^{2}(\omega)$ be an 1-periodic solution of

$$
\left\{\begin{aligned}
-\Delta \chi_{i k}^{j}+\frac{\partial}{\partial y_{j}} \pi_{2, i k} & =0 & & \text { in } \omega, \\
\frac{\partial}{\partial y_{j}} \chi_{i k}^{j} & =-W_{k}^{i}+\left|Y \backslash Y_{s}\right|^{-1} K_{k}^{i} & & \text { in } \omega, \\
\chi_{i k}^{j} & =0 & & \text { on } \partial \omega .
\end{aligned}\right.
$$


Since the compatibility condition,

$$
\int_{Y \backslash Y_{s}}\left\{-W_{k}^{i}+\left|Y \backslash Y_{s}\right|^{-1} K_{k}^{i}\right\} d y=0
$$

is satisfied, the 1-periodic solutions of (6.3) exist. Moreover, under the assumption that $\partial Y_{s}$ is $C^{1, \alpha}$, the functions $\nabla \chi_{i k}^{j}$ and $\pi_{2, i k}$ are bounded. As usual, we extend $\chi_{i k}^{j}$ from $\omega$ to $\mathbb{R}^{d}$ by zero. Fix a function $\varphi \in C_{0}^{\infty}(B(0,1 / 8))$ with the properties that $\varphi \geq 0$ and $\int_{\mathbb{R}^{d}} \varphi d x=1$. Let

$$
S_{\varepsilon}(\psi)(x)=\psi * \varphi_{\varepsilon}(x)=\int_{\mathbb{R}^{d}} \psi(y) \varphi_{\varepsilon}(x-y) d y,
$$

where $\varphi_{\varepsilon}(x)=\varepsilon^{-d} \varphi(x / \varepsilon)$. Define $\Phi_{\varepsilon}(x)=\left(\Phi_{\varepsilon}^{1}(x), \Phi_{\varepsilon}^{2}(x), \ldots, \Phi_{\varepsilon}^{d}(x)\right)$, where

$$
\Phi_{\varepsilon}^{j}(x)=\varepsilon \eta_{\varepsilon}(x) \chi_{k \ell}^{j}(x / \varepsilon) \frac{\partial}{\partial x_{\ell}} S_{\varepsilon}\left(f_{k}-\frac{\partial p_{0}}{\partial x_{k}}\right),
$$

$p_{0}$ is a solution of the Neumann problem (4.12), and $\eta_{\varepsilon}$ is a cut-off function in $C_{0}^{1}(\Omega)$ such that $0 \leq \eta_{\varepsilon} \leq 1, \eta_{\varepsilon}=1$ in $\Omega \backslash \Sigma_{3 d \varepsilon}, \eta_{\varepsilon}=0$ in $\Sigma_{2 d \varepsilon}$, and $\left|\nabla \eta_{\varepsilon}\right| \leq C \varepsilon^{-1}$. The use of the $\varepsilon$-smoothing operator $S_{\varepsilon}$ in (6.5) allows us to trade excessive powers of $\varepsilon$ for lowering derivatives of $f-\nabla p_{0}$.

The following lemma will be useful to us.

Lemma 6.2. Let $S_{\varepsilon}$ be defined by (6.4). Then

$$
\left\|\psi-\eta_{\varepsilon} S_{\varepsilon}(\psi)\right\|_{L^{2}(\Omega)} \leq C\|\psi\|_{L^{2}\left(\Sigma_{3 d \varepsilon}\right)}+C \varepsilon\|\nabla \psi\|_{L^{2}\left(\Omega \backslash \Sigma_{d \varepsilon}\right)}
$$

for $0<\varepsilon<1$.

Proof. Note that

$$
\left\|\psi-\eta_{\varepsilon} S_{\varepsilon}(\psi)\right\|_{L^{2}(\Omega)} \leq\left\|\left(1-\eta_{\varepsilon}\right) \psi\right\|_{L^{2}(\Omega)}+\| \eta_{\varepsilon}\left(\psi-S_{\varepsilon}(\psi) \|_{L^{2}(\Omega)} .\right.
$$

Clearly, the first term in the right-side hand is bounded by $\|\psi\|_{L^{2}\left(\Sigma_{3 d \varepsilon}\right)}$. To bound the second term, we use

$$
\psi(x)-S_{\varepsilon}(\psi)(x)=\int_{\mathbb{R}^{d}} \varphi_{\varepsilon}(y)[\psi(x-y)-\psi(x)] d y
$$

and

$$
\psi(x-y)-\psi(x)=\int_{0}^{1}(-y) \cdot \nabla \psi(x-t y) d t
$$

It follows that

$$
\begin{aligned}
\| \eta_{\varepsilon}\left(\psi-S_{\varepsilon}(\psi) \|_{L^{2}(\Omega)}\right. & \leq \int_{\mathbb{R}^{d}} \varphi_{\varepsilon}(y)\|\psi(\cdot-y)-\psi(\cdot)\|_{L^{2}\left(\Omega \backslash \Sigma_{2 d \varepsilon}\right)} d y \\
& \leq C \int_{\mathbb{R}^{d}} \varphi_{\varepsilon}(y)|y| d y\|\nabla \psi\|_{L^{2}\left(\Omega \backslash \Sigma_{d \varepsilon}\right)} \\
& \leq C \varepsilon\|\nabla \psi\|_{L^{2}\left(\Omega \backslash \Sigma_{d \varepsilon}\right)},
\end{aligned}
$$

where we have used Minkowski's inequality. 
Note that for $x \in \Omega_{\varepsilon}$,

$$
\begin{gathered}
\operatorname{div}\left(\Phi_{\varepsilon}\right)=\operatorname{div}\left(\chi_{k \ell}\right)(x / \varepsilon)\left[\eta_{\varepsilon} \frac{\partial}{\partial x_{\ell}} S_{\varepsilon}\left(f_{k}-\frac{\partial p_{0}}{\partial x_{k}}\right)\right]+\varepsilon \chi_{k \ell}^{j}(x / \varepsilon) \frac{\partial}{\partial x_{j}}\left[\eta_{\varepsilon} \frac{\partial}{\partial x_{\ell}} S_{\varepsilon}\left(f_{k}-\frac{\partial p_{0}}{\partial x_{k}}\right)\right] \\
=-\left[W_{k}^{\ell}(x / \varepsilon)-f_{Y \backslash Y_{s}} W_{k}^{\ell}\right]\left[\eta_{\varepsilon} \frac{\partial}{\partial x_{\ell}} S_{\varepsilon}\left(f_{k}-\frac{\partial p_{0}}{\partial x_{k}}\right)\right] \\
+\varepsilon \chi_{k \ell}^{j}(x / \varepsilon) \frac{\partial}{\partial x_{j}}\left[\eta_{\varepsilon} \frac{\partial}{\partial x_{\ell}} S_{\varepsilon}\left(f_{k}-\frac{\partial p_{0}}{\partial x_{k}}\right)\right] .
\end{gathered}
$$

Since

$$
\begin{aligned}
\operatorname{div}\left(W(x / \varepsilon)\left(f-\nabla p_{0}\right)\right) & =W_{k}^{\ell}(x / \varepsilon) \frac{\partial}{\partial x_{\ell}}\left(f_{k}-\frac{\partial p_{0}}{\partial x_{k}}\right) \\
& =\left[W_{k}^{\ell}(x / \varepsilon)-f_{Y \backslash Y_{s}} W_{k}^{\ell}\right] \frac{\partial}{\partial x_{\ell}}\left(f_{k}-\frac{\partial p_{0}}{\partial x_{k}}\right)
\end{aligned}
$$

where we have used the equation in (4.12), it follows that

$$
\begin{aligned}
& \left\|\operatorname{div}\left(\Phi_{\varepsilon}+W(x / \varepsilon)\left(f-\nabla p_{0}\right)\right)\right\|_{L^{2}\left(\Omega_{d \varepsilon}\right)} \\
& \leq C\left\|\nabla\left(f-\nabla p_{0}\right)\right\|_{L^{2}\left(\Sigma_{3 d \varepsilon}\right)}+C\left\|\nabla\left[\left(f-\nabla p_{0}\right)-S_{\varepsilon}\left(f-\nabla p_{0}\right)\right]\right\|_{L^{2}\left(\Omega \backslash \Sigma_{2 d \varepsilon}\right)} \\
& \quad+C \varepsilon\left\|\nabla^{2} S_{\varepsilon}\left(f-\nabla p_{0}\right)\right\|_{L^{2}\left(\Omega \backslash \Sigma_{2 d \varepsilon}\right)} .
\end{aligned}
$$

Let $\left(u_{\varepsilon}, p_{\varepsilon}\right)$ be a weak solution of (6.1) with $\int_{\Omega_{\varepsilon}} p_{\varepsilon} d x=0$. Let

$$
v_{\varepsilon}=u_{\varepsilon}-\left\{W(x / \varepsilon)\left(f-\nabla p_{0}\right)+\Phi_{\varepsilon}+\Psi_{t}+\Psi_{n}\right\},
$$

where $\Phi_{\varepsilon}$ is defined by (6.5), and $\Psi_{t}, \Psi_{n}$ are given by Theorems 4.4 and 5.7, respectively. Using (2.20), a direct computation shows that

$$
\begin{aligned}
& -\varepsilon^{2} \Delta\left\{W(x / \varepsilon)\left(f-\nabla p_{0}\right)\right\}+\nabla\left\{p_{0}+\varepsilon \pi(x / \varepsilon)\left(f-\nabla p_{0}\right)\right\} \\
& =f-\varepsilon^{2} \nabla\left(W(x / \varepsilon) \nabla\left(f-\nabla p_{0}\right)\right)-\varepsilon(\nabla W)(x / \varepsilon) \cdot \nabla\left(f-\nabla p_{0}\right)+\varepsilon \pi(x / \varepsilon) \nabla\left(f-\nabla p_{0}\right) \\
& \quad+\sigma_{\varepsilon}\left(f-\nabla p_{0}\right)
\end{aligned}
$$

in $\Omega_{\varepsilon}$, where $\sigma_{\varepsilon}$ is given by (2.21). It follows that

$$
\begin{aligned}
-\varepsilon^{2} \Delta v_{\varepsilon} & +\nabla\left\{p_{\varepsilon}-p_{0}-p_{t}-p_{n}-\varepsilon \pi(x / \varepsilon)\left(f-\nabla p_{0}\right)\right\} \\
=\varepsilon^{2} \Delta \Phi_{\varepsilon} & +\varepsilon^{2} \nabla\left(W(x / \varepsilon) \nabla\left(f-\nabla p_{0}\right)\right)-\sigma_{\varepsilon}\left(f-\nabla p_{0}\right) \\
& +\varepsilon(\nabla W)(x / \varepsilon) \cdot \nabla\left(f-\nabla p_{0}\right)-\varepsilon \pi(x / \varepsilon) \nabla\left(f-\nabla p_{0}\right)
\end{aligned}
$$

in $\Omega_{\varepsilon}$. Also, observe that

$$
\operatorname{div}\left(v_{\varepsilon}\right)=-\operatorname{div}\left(\Phi_{\varepsilon}+W(x / \varepsilon)\left(f-\nabla p_{0}\right)\right) \quad \text { in } \Omega_{\varepsilon},
$$

$v_{\varepsilon}=0$ on $\Gamma_{\varepsilon}$, and that

$$
v_{\varepsilon}=\gamma n \quad \text { on } \partial \Omega
$$


where $\gamma$ is a constant satisfying (5.25). Hence, by Theorem 3.1 as well as Remark 3.2 and the estimate (2.22) for $\sigma_{\varepsilon}$,

$$
\begin{aligned}
& \varepsilon\left\|\nabla v_{\varepsilon}\right\|_{L^{2}\left(\Omega_{\varepsilon}\right)}+\left\|v_{\varepsilon}\right\|_{L^{2}\left(\Omega_{\varepsilon}\right)} \\
& \leq C\left\{\varepsilon\left\|\nabla \Phi_{\varepsilon}\right\|_{L^{2}\left(\Omega_{\varepsilon}\right)}+\varepsilon\left\|\nabla\left(f-\nabla p_{0}\right)\right\|_{L^{2}(\Omega)}+\left\|f-\nabla p_{0}\right\|_{L^{2}\left(\Sigma_{c \varepsilon}\right)}+\left\|\operatorname{div}\left(v_{\varepsilon}\right)\right\|_{L^{2}\left(\Omega_{\varepsilon}\right)}+|\gamma|\right\} \\
& \leq C\left\{\varepsilon\left\|\nabla\left(f-\nabla p_{0}\right)\right\|_{L^{2}(\Omega)}+\left\|\nabla\left(f-\nabla p_{0}\right)\right\|_{L^{2}\left(\Sigma_{3 d \varepsilon}\right)}+\left\|f-\nabla p_{0}\right\|_{L^{2}\left(\Sigma_{c \varepsilon}\right)}\right. \\
& \quad+C\left\|\nabla\left[\left(f-\nabla p_{0}\right)-S_{\varepsilon}\left(f-\nabla p_{0}\right)\right]\right\|_{L^{2}\left(\Omega \backslash \Sigma_{2 d \varepsilon}\right)} \\
& \left.\quad+\varepsilon\left\|\nabla^{2} S_{\varepsilon}\left(f-\nabla p_{0}\right)\right\|_{L^{2}\left(\Omega \backslash \Sigma_{2 d \varepsilon}\right)}+\varepsilon\left\|\nabla_{\tan }\left(f-\nabla p_{0}\right)\right\|_{L^{2}(\partial \Omega)}\right\},
\end{aligned}
$$

where we have used (6.7) and (5.25). Let

$$
q_{\varepsilon}=p_{\varepsilon}-p_{0}-q_{t}-q_{n}-\varepsilon \pi(x / \varepsilon)\left(f-\nabla p_{0}\right) .
$$

Note that Theorem 3.1 also gives

$$
\begin{aligned}
\left\|q_{\varepsilon}-f_{\Omega_{\varepsilon}} q_{\varepsilon}\right\|_{L^{2}\left(\Omega_{\varepsilon}\right)} \leq C & \left\{\left\|\nabla\left(f-\nabla p_{0}\right)\right\|_{L^{2}(\Omega)}+\left\|\nabla\left(f-\nabla p_{0}\right)\right\|_{L^{2}\left(\Sigma_{3 d \varepsilon}\right)}\right. \\
& +\left\|f-\nabla p_{0}\right\|_{L^{2}\left(\Sigma_{c \varepsilon}\right)} \\
& +C\left\|\nabla\left[\left(f-\nabla p_{0}\right)-S_{\varepsilon}\left(f-\nabla p_{0}\right)\right]\right\|_{L^{2}\left(\Omega \backslash \Sigma_{2 d \varepsilon}\right)} \\
& \left.+\varepsilon\left\|\nabla^{2} S_{\varepsilon}\left(f-\nabla p_{0}\right)\right\|_{L^{2}\left(\Omega \backslash \Sigma_{2 d \varepsilon}\right)}+\varepsilon\left\|\nabla_{\tan }\left(f-\nabla p_{0}\right)\right\|_{L^{2}(\partial \Omega)}\right\} .
\end{aligned}
$$

Lemma 6.3. Let $\left(u_{\varepsilon}, p_{\varepsilon}\right)$ be a weak solution of (6.1) with $\int_{\Omega_{\varepsilon}} p_{\varepsilon} d x=0$. Then

$$
\begin{aligned}
& \varepsilon\left\|\nabla\left(u_{\varepsilon}-W(x / \varepsilon)\left(f-\nabla p_{0}\right)\right)\right\|_{L^{2}\left(\Omega_{\varepsilon}\right)}+\left\|p_{\varepsilon}-p_{0}\right\|_{L^{2}\left(\Omega_{\varepsilon}\right)} \\
& \leq C \varepsilon^{1 / 2}\left\{\begin{array}{l}
\varepsilon^{1 / 2}\left\|\nabla\left(f-\nabla p_{0}\right)\right\|_{L^{2}(\Omega)}+\varepsilon^{-1 / 2}\left\|\nabla\left(f-\nabla p_{0}\right)\right\|_{L^{2}\left(\Sigma_{3 d \varepsilon}\right)}+\varepsilon^{-1 / 2}\left\|f-\nabla p_{0}\right\|_{L^{2}\left(\Sigma_{c \varepsilon}\right)} \\
\quad+\varepsilon^{-1 / 2}\left\|\nabla\left[\left(f-\nabla p_{0}\right)-S_{\varepsilon}\left(f-\nabla p_{0}\right)\right]\right\|_{L^{2}\left(\Omega \backslash \Sigma_{2 d \varepsilon}\right)} \\
\quad+\varepsilon^{1 / 2}\left\|\nabla^{2} S_{\varepsilon}\left(f-\nabla p_{0}\right)\right\|_{L^{2}\left(\Omega \backslash \Sigma_{2 d \varepsilon}\right)}+\left\|f-\nabla p_{0}\right\|_{L^{2}(\partial \Omega)} \\
\left.\quad+\|b\|_{L^{2}(\partial \Omega)}+\varepsilon\left\|\nabla_{\tan } b\right\|_{L^{2}(\partial \Omega)}+\sqrt{\varepsilon}\left\|\nabla_{\tan }\left(f-\nabla p_{0}\right)\right\|_{L^{2}(\partial \Omega)}\right\}
\end{array}\right.
\end{aligned}
$$

for $0<\varepsilon<1$.

Proof. The estimate (6.11) follows readily from (6.9), (6.10), (4.13), and (5.24).

To bound the right-hand side of (6.11), we let $p_{0}=p_{0}^{(1)}+p_{0}^{(2)}$, where $p_{0}^{(1)}$ and $p_{0}^{(2)}$ are solutions of the Neumann problems,

$$
\left\{\begin{aligned}
K_{j}^{i} \frac{\partial}{\partial x_{i}}\left(f_{j}-\frac{\partial p_{0}^{(1)}}{\partial x_{j}}\right)=0 & \text { in } \Omega \\
n_{i} K_{j}^{i}\left(f_{j}-\frac{\partial p_{0}^{(1)}}{\partial x_{j}}\right)=0 & \text { on } \partial \Omega
\end{aligned}\right.
$$

and

$$
\begin{cases}K_{j}^{i} \frac{\partial^{2} p_{0}^{(2)}}{\partial x_{i} \partial x_{j}}=0 & \text { in } \Omega, \\ n_{i} K_{j}^{i} \frac{\partial p_{0}^{(2)}}{\partial x_{j}}=-b \cdot n & \text { on } \partial \Omega,\end{cases}
$$

respectively, with $\int_{\Omega} p_{0}^{(1)} d x=\int_{\Omega} p_{0}^{(2)} d x=0$. 
Lemma 6.4. Let $p_{0}^{(1)}$ be a solution of (6.12) for some $f \in C^{1,1 / 2}\left(\bar{\Omega}, \mathbb{R}^{d}\right)$. Then

$$
\begin{gathered}
\left\|\nabla p_{0}^{(1)}\right\|_{L^{\infty}(\Omega)}+\left\|\nabla^{2} p_{0}^{(1)}\right\|_{L^{\infty}(\Omega)} \leq C\|f\|_{C^{1,1 / 2}(\bar{\Omega})}, \\
\left\|\nabla^{2} S_{\varepsilon}(f)\right\|_{L^{\infty}\left(\Omega \backslash \Sigma_{\varepsilon}\right)}+\left\|\nabla^{2} S_{\varepsilon}\left(\nabla p_{0}^{(1)}\right)\right\|_{L^{\infty}\left(\Omega \backslash \Sigma_{\varepsilon}\right)} \leq C \varepsilon^{-1 / 2}\|f\|_{C^{1,1 / 2}(\bar{\Omega})},
\end{gathered}
$$

and

$$
\left\|\nabla f-S_{\varepsilon}(\nabla f)\right\|_{L^{\infty}\left(\Omega \backslash \Sigma_{\varepsilon}\right)}+\left\|\nabla^{2} p_{0}^{(1)}-S_{\varepsilon}\left(\nabla^{2} p_{0}^{(1)}\right)\right\|_{L^{\infty}\left(\Omega \backslash \Sigma_{\varepsilon}\right)} \leq C \varepsilon^{1 / 2}\|f\|_{C^{1,1 / 2}(\bar{\Omega})} .
$$

Proof. Since $\Omega$ is a bounded $C^{2, \alpha}$ domain, the first inequality in (6.14) follows from the classical $C^{2}$ estimates, up to the boundary, for second-order elliptic equations with constant coefficients. Next, note that for $x \in \Omega \backslash \Sigma_{\varepsilon}$,

$$
\begin{aligned}
\frac{\partial}{\partial x_{i}} S_{\varepsilon}(\nabla f)(x) & =\varepsilon^{-1-d} \int_{\mathbb{R}^{d}} \frac{\partial \varphi}{\partial y_{i}}(y / \varepsilon) \nabla f(x-y) d y \\
& =\varepsilon^{-1-d} \int_{\mathbb{R}^{d}} \frac{\partial \varphi}{\partial y_{i}}(y / \varepsilon)[\nabla f(x-y)-\nabla f(x)] d y .
\end{aligned}
$$

It follows that

$$
\begin{aligned}
\left\|\nabla^{2} S_{\varepsilon}(f)\right\|_{L^{\infty}\left(\Omega \backslash \Sigma_{\varepsilon}\right)} & \leq C \varepsilon^{-1-d} \int_{B(0, \varepsilon / 4)}\left|\nabla \varphi(y / \varepsilon)\left\|\left.y\right|^{1 / 2} d y\right\| f \|_{C^{1,1 / 2}(\bar{\Omega})}\right. \\
& \leq C \varepsilon^{-1 / 2}\|f\|_{C^{1,1 / 2}(\bar{\Omega})} .
\end{aligned}
$$

By the interior $C^{2,1 / 2}$ estimates,

$$
\left|\nabla^{2} p_{0}^{(1)}(x-y)-\nabla^{2} p_{0}^{(1)}(y)\right| \leq C|y|^{1 / 2}\left\{\|f\|_{C^{1,1 / 2}(\bar{\Omega})}+\left\|p_{0}^{(1)}\right\|_{C^{2}(\bar{\Omega})}\right\}
$$

for any $x \in \Omega \backslash \Sigma_{\varepsilon}$ and $|y| \leq \frac{1}{4} \varepsilon$. As in the case of $\nabla^{2} S_{\varepsilon}(f)$, this implies that

$$
\left|\nabla^{2} S_{\varepsilon}\left(\nabla p_{0}^{(1)}\right)(x)\right| \leq C \varepsilon^{-1 / 2}\|f\|_{C^{1,1 / 2}(\bar{\Omega})}
$$

for any $x \in \Omega \backslash \Sigma_{\varepsilon}$. Finally, to see (6.15), we write

$$
S_{\varepsilon}(\nabla f)(x)-\nabla f(x)=\int_{\mathbb{R}^{d}} \varphi_{\varepsilon}(x-y)[\nabla f(x-y)-\nabla f(x)] d y
$$

and proceed as in the previous estimates.

Lemma 6.5. Let $p_{0}^{(2)}$ be a solution of (6.13). Then

$$
\begin{aligned}
&\left\|\nabla p_{0}^{(2)}\right\|_{L^{2}(\Omega)}+\left\|\nabla p_{0}^{(2)}\right\|_{L^{2}(\partial \Omega)}+\varepsilon^{-1 / 2}\left\|\nabla p_{0}\right\|_{L^{2}\left(\Sigma_{\varepsilon}\right)} \leq C\|b \cdot n\|_{L^{2}(\partial \Omega)}, \\
&\left\|\nabla^{2} p_{0}^{(2)}\right\|_{L^{2}(\Omega)}+\left\|\nabla^{2} p_{0}^{(2)}\right\|_{L^{2}(\partial \Omega)}+\varepsilon^{-1 / 2}\left\|\nabla^{2} p_{0}^{(2)}\right\|_{L^{2}\left(\Sigma_{\varepsilon}\right)} \leq C\|b \cdot n\|_{H^{1}(\partial \Omega)}, \\
& \varepsilon^{-1 / 2}\left\|\nabla^{2} p_{0}^{(2)}-S_{\varepsilon}\left(\nabla^{2} p_{0}^{(2)}\right)\right\|_{L^{2}\left(\Omega \backslash \Sigma_{2 \varepsilon}\right)} \leq C\|b \cdot n\|_{H^{1}(\partial \Omega)}, \\
& \varepsilon^{1 / 2}\left\|\nabla^{2} S_{\varepsilon}\left(\nabla p_{0}^{(2)}\right)\right\|_{L^{2}\left(\Omega \backslash \Sigma_{2 \varepsilon}\right)} \leq C\|b \cdot n\|_{H^{1}(\partial \Omega)},
\end{aligned}
$$

for $0<\varepsilon<1$. 
Proof. The estimates (6.16)-(6.19) follow from the nontangential-maximal-function and squarefunction estimates for the Neumann problems,

$$
\begin{gathered}
\left\|\left(\nabla p_{0}^{(2)}\right)^{*}\right\|_{L^{2}(\partial \Omega)}+\left(\int_{\Omega} \operatorname{dist}(x, \partial \Omega)\left|\nabla^{2} p_{0}^{(2)}(x)\right|^{2} d x\right)^{1 / 2} \leq C\|b \cdot n\|_{L^{2}(\partial \Omega)} \\
\left\|\left(\nabla^{2} p_{0}^{(2)}\right)^{*}\right\|_{L^{2}(\partial \Omega)}+\left(\int_{\Omega} \operatorname{dist}(x, \partial \Omega)\left|\nabla^{3} p_{0}^{(2)}(x)\right|^{2} d x\right)^{1 / 2} \leq C\|b \cdot n\|_{H^{1}(\partial \Omega)},
\end{gathered}
$$

where $(u)^{*}$ denotes the nontangential maximal function of $u$, defined by (5.15). We remark that the estimate (6.20) hols if $\Omega$ is a bounded Lipschitz domain [12, while (6.21) holds for $C^{2, \alpha}$ domains.

We only give the proof of (6.18); the others follow readily from (6.20)-(6.21). Choose $\widetilde{\eta}_{\varepsilon} \in C_{0}^{1}(\Omega)$ such that $\widetilde{\eta}_{\varepsilon}=1$ in $\Omega \backslash \Sigma_{2 \varepsilon}, \widetilde{\eta}_{\varepsilon}=0$ in $\Sigma_{\varepsilon}$, and $\left|\nabla \widetilde{\eta}_{\varepsilon}\right| \leq C \varepsilon^{-1}$. Then the left-hand side of (6.18) is bounded by

$$
\varepsilon^{-1 / 2}\left\|\nabla^{2} p_{0}^{(2)}-\widetilde{\eta}_{\varepsilon} S_{\varepsilon}\left(\nabla^{2} p_{0}^{(2)}\right)\right\|_{L^{2}(\Omega)} .
$$

Using the same argument as in the proof of (6.6), we may show that (6.22) is bounded by

$$
C \varepsilon^{-1 / 2}\left\|\nabla^{2} p_{0}^{(2)}\right\|_{L^{2}\left(\Sigma_{3 \varepsilon}\right)}+C \varepsilon^{1 / 2}\left\|\nabla^{3} p_{0}^{(2)}\right\|_{L^{2}\left(\Omega \backslash \Sigma_{\varepsilon}\right)} \leq C\|b \cdot n\|_{H^{1}(\partial \Omega)},
$$

where we have used (6.21) for the last step.

We are now in a position to give the proof of Theorem 6.1.

Proof of Theorem 6.1. Using Lemmas 6.4 and 6.5. it is not hard to see that the right-hand side of (6.11) is bounded by

$$
C \sqrt{\varepsilon}\left\{\|f\|_{C^{1,1 / 2}(\Omega)}+\|b \cdot n\|_{H^{1}(\partial \Omega)}+\|b\|_{L^{2}(\partial \Omega)}+\varepsilon\left\|\nabla_{\tan } b\right\|_{L^{2}(\partial \Omega)}\right\} .
$$

As a result, we have proved that

$$
\begin{aligned}
\varepsilon \| \nabla\left(u_{\varepsilon}-\right. & \left.W(x / \varepsilon)\left(f-\nabla p_{0}\right)\right)\left\|_{L^{2}\left(\Omega_{\varepsilon}\right)}+\right\| p_{\varepsilon}-p_{0} \|_{L^{2}\left(\Omega_{\varepsilon}\right)} \\
& \leq C \sqrt{\varepsilon}\left\{\|f\|_{C^{1,1 / 2}(\Omega)}+\|b \cdot n\|_{H^{1}(\partial \Omega)}+\|b\|_{L^{2}(\partial \Omega)}+\varepsilon\left\|\nabla_{\tan } b\right\|_{L^{2}(\partial \Omega)}\right\} .
\end{aligned}
$$

In view of Lemma 2.2, it remains to show that

$$
\left\|P_{\varepsilon}-p_{0}\right\|_{L^{2}(\Omega)} \leq C \sqrt{\varepsilon}\left\{\|f\|_{C^{1,1 / 2}(\Omega)}+\|b \cdot n\|_{H^{1}(\partial \Omega)}+\|b\|_{L^{2}(\partial \Omega)}+\varepsilon\left\|\nabla_{\tan } b\right\|_{L^{2}(\partial \Omega)}\right\},
$$

where $P_{\varepsilon}$ is an extension of $p_{\varepsilon}$ to $\Omega$, defined by (2.6). To this end, we define

$$
p_{0}^{\varepsilon}= \begin{cases}p_{0} & \text { if } x \in \Omega_{\varepsilon}, \\ f_{\varepsilon\left(Y_{f}+z_{k}\right)} p_{0} & \text { if } x \in \varepsilon\left(Y_{s}+z_{k}\right) \text { and } \varepsilon\left(Y+z_{k}\right) \subset \Omega \text { for some } z_{k} \in \mathbb{Z}^{d},\end{cases}
$$

i.e., we extend $\left.p_{0}\right|_{\Omega_{\varepsilon}}$ to $\Omega$ in the same manner as we do $p_{\varepsilon}$ from $\Omega_{\varepsilon}$ to $\Omega$. Then,

$$
\begin{aligned}
\left\|P_{\varepsilon}-p_{0}\right\|_{L^{2}(\Omega)} & \leq\left\|P_{\varepsilon}-p_{0}^{\varepsilon}\right\|_{L^{2}(\Omega)}+\left\|p_{0}^{\varepsilon}-p_{0}\right\|_{L^{2}(\Omega)} \\
& =\left\|p_{\varepsilon}-p_{0}\right\|_{L^{2}\left(\Omega_{\varepsilon}\right)}+\left\|P_{\varepsilon}-p_{0}^{\varepsilon}\right\|_{L^{2}\left(\Omega \backslash \Omega_{\varepsilon}\right)}+\left\|p_{0}^{\varepsilon}-p_{0}\right\|_{L^{2}\left(\Omega \backslash \Omega_{\varepsilon}\right)} .
\end{aligned}
$$

Note that

$$
\left\|P_{\varepsilon}-p_{0}^{\varepsilon}\right\|_{L^{2}\left(\Omega \backslash \Omega_{\varepsilon}\right)} \leq C\left\|p_{\varepsilon}-p_{0}\right\|_{L^{2}\left(\Omega_{\varepsilon}\right)} .
$$


Using Poincaré's inequality on each cell $\varepsilon\left(Y_{f}+z_{k}\right)$, we may show that

$$
\left\|p_{0}^{\varepsilon}-p_{0}\right\|_{L^{2}\left(\Omega \backslash \Omega_{\varepsilon}\right)} \leq C \varepsilon\left\|\nabla p_{0}\right\|_{L^{2}(\Omega)} \leq C \varepsilon\left\{\|f\|_{L^{2}(\Omega)}+\|b\|_{L^{2}(\partial \Omega)}\right\} .
$$

As a result, we have proved that

$$
\left\|P_{\varepsilon}-p_{0}\right\|_{L^{2}(\Omega)} \leq C\left\|p_{\varepsilon}-p_{0}\right\|_{L^{2}\left(\Omega_{\varepsilon}\right)}+C \varepsilon\left\{\|f\|_{L^{2}(\Omega)}+\|b\|_{L^{2}(\partial \Omega)}\right\} .
$$

This completes the proof.

Remark 6.6. Let $u(x, x / \varepsilon)$ be given by (1.6). Due to the discrepancy of $u_{\varepsilon}$ and $u(x, x / \varepsilon)$ on $\partial \Omega$, the $O(\sqrt{\varepsilon})$ rate in Theorem 1.1 is sharp. Indeed, by applying the following trace inequality to the function $v=v_{\varepsilon}=u_{\varepsilon}-u(x, x / \varepsilon)$,

$$
\|v\|_{L^{2}(\partial \Omega)} \leq C\left\{\varepsilon^{-1 / 2}\|v\|_{L^{2}\left(\Sigma_{c \varepsilon}\right)}+\|v\|_{L^{2}\left(\Sigma_{c \varepsilon}\right)}^{1 / 2}\|\nabla v\|_{L^{2}\left(\Sigma_{c \varepsilon}\right)}^{1 / 2}\right\}
$$

we obtain

$$
\begin{aligned}
\sqrt{\varepsilon}\left\|v_{\varepsilon}\right\|_{L^{2}(\partial \Omega)} & \leq C\left\{\left\|v_{\varepsilon}\right\|_{L^{2}\left(\Omega_{\varepsilon}\right)}+\varepsilon\left\|\nabla v_{\varepsilon}\right\|_{L^{2}\left(\Omega_{\varepsilon}\right)}\right\} \\
& \leq C \varepsilon\left\|\nabla v_{\varepsilon}\right\|_{L^{2}\left(\Omega_{\varepsilon}\right)},
\end{aligned}
$$

where we have used the Cauchy inequality for the first inequality and (2.9) for the second. It follows that the error estimate

$$
\varepsilon\left\|\nabla v_{\varepsilon}\right\|_{L^{2}\left(\Omega_{\varepsilon}\right)}=o(\sqrt{\varepsilon}) \quad \text { as } \varepsilon \rightarrow 0,
$$

cannot hold in general. In fact, if $\Omega$ is smooth and uniformly convex, then

$$
\begin{aligned}
\lim _{\varepsilon \rightarrow 0} f_{\partial \Omega}\left|v_{\varepsilon}\right|^{2} d \sigma & =\lim _{\varepsilon \rightarrow 0} f_{\partial \Omega}\left|W(x / \varepsilon)\left(f-\nabla p_{0}\right)\right|^{2} d \sigma \\
& =f_{\partial \Omega}\left|K\left(f-\nabla p_{0}\right)\right|^{2} d \sigma .
\end{aligned}
$$

See the proof of Lemma 3.2 in [1]. Also, note that by Theorem 1.1,

$$
\left\|\nabla v_{\varepsilon}\right\|_{L^{2}\left(\Omega_{\varepsilon}\right)} \leq C \varepsilon^{-1 / 2}\|f\|_{C^{1,1 / 2}(\Omega)} .
$$

This, together with (6.26), yields

$$
\left\|v_{\varepsilon}\right\|_{L^{2}(\partial \Omega)} \leq C\left\{\varepsilon^{-1 / 2}\left\|v_{\varepsilon}\right\|_{L^{2}\left(\Omega_{\varepsilon}\right)}+\left(\varepsilon^{-1 / 2}\left\|v_{\varepsilon}\right\|_{2}\left(\Omega_{\varepsilon}\right)\right)^{1 / 2}\|f\|_{C^{1,1 / 2}(\Omega)}^{1 / 2}\right\} .
$$

As a result, it is not possible to have

$$
\left\|v_{\varepsilon}\right\|_{L^{2}\left(\Omega_{\varepsilon}\right)}=o(\sqrt{\varepsilon}) \quad \text { as } \varepsilon \rightarrow 0,
$$

unless $f=\nabla p_{0}$ in $\Omega$, in which case, $v_{\varepsilon} \equiv 0$ in $\Omega_{\varepsilon}$.

Finally, to see (6.26), choose a function $\beta \in C^{1}\left(\mathbb{R}^{d}, \mathbb{R}^{d}\right)$ such that $\beta \cdot n \geq c_{0}>0$ on $\partial \Omega$, $\operatorname{supp}(\beta) \subset\left\{x \in \mathbb{R}^{d}: \operatorname{dist}(x, \partial \Omega) \leq c \varepsilon\right\}$, and $|\nabla \beta| \leq C \varepsilon^{-1}$. It follows by the divergence theorem that

$$
\begin{aligned}
c_{0} \int_{\partial \Omega}|v|^{2} d \sigma & \leq \int_{\partial \Omega}|v|^{2} \beta \cdot n d \sigma \leq \int_{\Omega}|v|^{2} \operatorname{div}(\beta) d x+2 \int_{\Omega}|v\|\nabla v\| \beta| d x \\
& \leq C \varepsilon^{-1} \int_{\Sigma_{c \varepsilon}}|v|^{2} d x+C\|v\|_{L^{2}\left(\Sigma_{c \varepsilon}\right)}\|\nabla v\|_{L^{2}\left(\Sigma_{c \varepsilon}\right)}
\end{aligned}
$$

where we have used the Cauchy inequality for the last step. 


\section{References}

[1] H. Aleksanyan, H. Shahgholian, and P. Sjölin, Applications of Fourier analysis in homogenization of the Dirichlet problem: $L^{p}$ estimates, Arch. Ration. Mech. Anal. 215 (2015), no. 1, $65-87$.

[2] G. Allaire, Homogenization of the Stokes flow in a connected porous medium, Asymptotic Anal. 2 (1989), no. 3, 203-222.

[3] Continuity of the Darcy's law in the low-volume fraction limit, Ann. Scuola Norm. Sup. Pisa Cl. Sci. (4) 18 (1991), no. 4, 475-499.

[4] G. Allaire and A. Mikelić, One-phase Newtonian flow, Homogenization and porous media, Interdiscip. Appl. Math., vol. 6, Springer, New York, 1997, pp. 45-76, 259-275.

[5] G. A. Chechkin, A. L. Piatnitski, and A. S. Shamaev, Homogenization, Translations of Mathematical Monographs, vol. 234, American Mathematical Society, Providence, RI, 2007, Methods and applications, Translated from the 2007 Russian original by Tamara Rozhkovskaya.

[6] E. B. Fabes, C. E. Kenig, and G. C. Verchota, The Dirichlet problem for the Stokes system on Lipschitz domains, Duke Math. J. 57 (1988), no. 3, 769-793.

[7] S. Gu and J. Zhuge, Periodic homogenization of Green's functions for Stokes systems, Calc. Var. Partial Differential Equations 58 (2019), no. 3, Paper No. 114, 46.

[8] W. Jäger and A. Mikelić, On the flow conditions at the boundary between a porous medium and an impervious solid, Progress in partial differential equations: the Metz surveys, 3, Pitman Res. Notes Math. Ser., vol. 314, Longman Sci. Tech., Harlow, 1994, pp. 145-160.

[9] W. Jing, Layer potentials for Lamé systems and homogenization of perforated elastic medium with clamped holes, arXiv:2007.03333v2 (2020).

[10] _ A unified homogenization approach for the Dirichlet problem in perforated domains, SIAM J. Math. Anal. 52 (2020), no. 2, 1192-1220.

[11] H. Kacimi and F. Murat, Estimation de l'erreur dans des problèmes de Dirichlet où apparait un terme étrange, Partial differential equations and the calculus of variations, Vol. II, Progr. Nonlinear Differential Equations Appl., vol. 2, Birkhäuser Boston, Boston, MA, 1989, pp. 661696.

[12] C. E. Kenig, Harmonic analysis techniques for second order elliptic boundary value problems, CBMS Regional Conference Series in Mathematics, vol. 83, Published for the Conference Board of the Mathematical Sciences, Washington, DC; by the American Mathematical Society, Providence, RI, 1994.

[13] C. E. Kenig, F. Lin, and Z. Shen, Convergence rates in $L^{2}$ for elliptic homogenization problems, Arch. Ration. Mech. Anal. 203 (2012), no. 3, 1009-1036.

[14] J.-L. Lions, Asymptotic expansions in perforated media with a periodic structure, Rocky Mountain J. Math. 10 (1980), no. 1, 125-140.

[15] R. Lipton and M. Avellaneda, Darcy's law for slow viscous flow past a stationary array of bubbles, Proc. Roy. Soc. Edinburgh Sect. A 114 (1990), no. 1-2, 71-79. 
[16] Y. Lu, Homogenization of Stokes equations in perforated domains: a unified approach, J. Math. Fluid Mech. 22 (2020), no. 3, Art. 44, 13.

[17] E. Marušić-Paloka and A. Mikelić, An error estimate for correctors in the homogenization of the Stokes and the Navier-Stokes equations in a porous medium, Boll. Un. Mat. Ital. A (7) 10 (1996), no. 3, 661-671.

[18] _ The derivation of a nonlinear filtration law including the inertia effects via homogenization, Nonlinear Anal. 42 (2000), no. 1, Ser. A: Theory Methods, 97-137.

[19] N. Masmoudi, Some uniform elliptic estimates in a porous medium, C. R. Math. Acad. Sci. Paris 339 (2004), no. 12, 849-854.

[20] A. Mikelić, Homogenization of nonstationary Navier-Stokes equations in a domain with a grained boundary, Ann. Mat. Pura Appl. (4) 158 (1991), 167-179.

[21] D. Mitrea and I. Mitrea, On the regularity of Green functions in Lipschitz domains, Comm. Partial Differential Equations 36 (2011), no. 2, 304-327.

[22] O. A. Oleĭnik, A. S. Shamaev, and G. A. Yosifian, Mathematical problems in elasticity and homogenization, Studies in Mathematics and its Applications, vol. 26, North-Holland Publishing Co., Amsterdam, 1992.

[23] E. Sánchez-Palencia, Nonhomogeneous media and vibration theory, Lecture Notes in Physics, vol. 127, Springer-Verlag, Berlin-New York, 1980.

[24] R. Temam, Navier-Stokes equations, AMS Chelsea Publishing, Providence, RI, 2001, Theory and numerical analysis, Reprint of the 1984 edition.

Zhongwei Shen, Department of Mathematics, University of Kentucky, Lexington, Kentucky 40506, USA.

E-mail: zshen2@uky.edu 